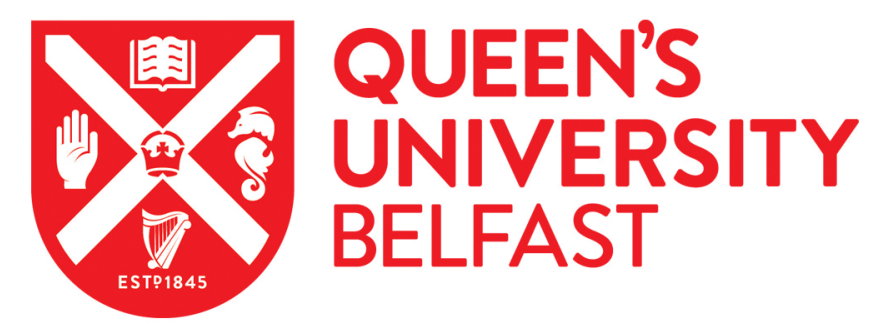

\title{
Review of metasurface antennas for computational microwave imaging
}

Imani, M. F., Gollub, J., Yurduseven, O., Diebold, A. V., Boyarsky, M., Fromenteze, T., Mancera, L-P.,

Sleasman, T., \& Smith, D. R. (2020). Review of metasurface antennas for computational microwave imaging. IEEE Transactions on Antennas and Propagation. https://doi.org/10.1109/TAP.2020.2968795

\author{
Published in: \\ IEEE Transactions on Antennas and Propagation
}

\section{Document Version:}

Peer reviewed version

\section{Queen's University Belfast - Research Portal:}

Link to publication record in Queen's University Belfast Research Portal

\section{Publisher rights}

(c) 2020 IEEE.

This work is made available online in accordance with the publisher's policies. Please refer to any applicable terms of use of the publisher.

\section{General rights}

Copyright for the publications made accessible via the Queen's University Belfast Research Portal is retained by the author(s) and / or other copyright owners and it is a condition of accessing these publications that users recognise and abide by the legal requirements associated with these rights.

Take down policy

The Research Portal is Queen's institutional repository that provides access to Queen's research output. Every effort has been made to ensure that content in the Research Portal does not infringe any person's rights, or applicable UK laws. If you discover content in the Research Portal that you believe breaches copyright or violates any law, please contact openaccess@qub.ac.uk. 


\title{
Review of metasurface antennas for computational microwave imaging
}

\author{
Mohammadreza F. Imani, Jonah Gollub, Okan Yurduseven, Senior Member, IEEE, Aaron V. Diebold, Michael \\ Boyarsky, Thomas Fromenteze, Laura Pulido-Mancera, Member, IEEE, Timothy Sleasman, Member, IEEE, and \\ David R. Smith, Senior Member, IEEE.
}

\begin{abstract}
This article covers recent advances in the fusion of metasurface antenna design and computational imaging concepts for the realization of imaging systems that are planar, fast, and low cost. We start by explaining the operation of metamaterial antennas which can generate diverse radiation patterns. Their advantages and distinctions from previous antennas are elucidated. We then provide an intuitive overview of the computational imaging framework and argue that metamaterial antennas are a near ideal platform for implementing such schemes at microwave frequencies. We describe two metamaterial antenna implementations: frequency-diverse and electronically reconfigurable. The trade-offs governing the design and operation of each architecture are examined. We conclude by examining the outlook of metamaterial antennas for microwave imaging and propose various future directions.
\end{abstract}

Index Terms-Metamaterials, Metasurfaces, Antennas, Computational Imaging, Microwave Imaging.

\section{INTRODUCTION}

$\mathbf{M}$ ICROWAVE imaging has been at the center of a wide swath of applications ranging from security screening [1] to earth and space observation [2]. A rich body of literature has examined both hardware and software aspects of microwave imaging, with many successful implementations being proposed and finding their utility in commercial applications. This growing interest is partially driven by the fact that microwaves can penetrate many optically opaque materials, such as clothing. Microwaves also have long wavelengths, such that they are non-ionizing and relatively safe for exposure to humans. This feature, despite providing lower resolution than optical or infrared approaches, is largely responsible for the ubiquity of microwave imaging and sensing. The long wavelength, however, is also the cause of the many complexities of implementing microwave imaging, as a very large aperture and the associated hardware are required to achieve desired (diffraction-limited) resolution. The common solutions are to mechanically scan an antenna [3], [4] to synthesize a large aperture, or to employ a large multi-static array [5]-[10], or a combination of both [1], [11]. Either method can achieve excellent imaging performance, exemplified by their widespread use in real-life applications such as airport

M. F. Imani, J. Gollub, A. V. Diebold, M. Boyarsky, T. Sleasman, L. PulidoMancera, D. R. Smith are with the Department of Electrical and Computer Engineering, Duke University, Durham, NC 27708, USA.

T. Fromenteze is with the University of Limoges, XLIM, UMR 7252, F87000 Limoges, France.

O. Yurduseven is with the School of Electronics, Electrical Engineering and Computer Science, Queen's University Belfast, Belfast BT3 9DT, U.K. screening systems. However, they come at the cost of large bulky systems, slow acquisition, high power consumption, or prohibitively high cost. These challenges restrict microwave imaging applications to scenarios with minimal economic or speed constraints (e.g. government-funded surveillance). To improve upon the conventional solutions demands a new perspective. In this paper, we review a recent solution to this need that is based on the fusion of two innovative concepts: metamaterials and computational imaging (CI) techniques.

Over the last two decades, metamaterials and metasurfaces have spurred numerous innovative antenna designs and revamped established techniques. In particular, they offer a more cost-effective solution to pattern synthesis compared to conventional phased arrays, which rely on active phase shifters and/or complex feed networks [12]. Metasurface antennas have proven to be economical and are beginning to fulfill massmarket needs for pattern synthesis in satellite communication [13], radar systems, and telecommunication base stations [14].

In parallel, another innovation that has spurred a departure from classical methodologies is CI [15]-[17], entailing the joint design of hardware and software of an imaging system to improve cost, complexity, capability or speed. This notion has especially been the subject of great attention at optical frequencies. An overarching theme in this framework is to forego the one-to-one relationship between each point in the scene and the reconstructed image, as is the case in most conventional cameras, and instead use illumination patterns that can multiplex the information content of the entire scene under test into a few simple measurements, and leverage ever growing computational power to form the image using computational techniques. In this paradigm-shifting approach, the burden of image formation is transferred from hardware to software. A plethora of work has been dedicated to this topic, with lensless imaging [18], compressive imaging [19], [20], ptychography [21], imaging through scattering medium [22]-[25], and computational ghost imaging [26], [27] being some of the prominent examples.

Transposing CI methodologies to the microwave regime can lead to a radical shift; instead of antennas with welldefined beams scanning a portion of a scene of interest, the whole scene is illuminated by spatially diverse patterns and its information is encoded into a few measurements, as visualized in Fig. 1. In this framework, the requirement for the antenna hardware is drastically different from conventional schemesit needs to generate spatially diverse patterns and alter them to multiplex the information (as opposed to well-defined 

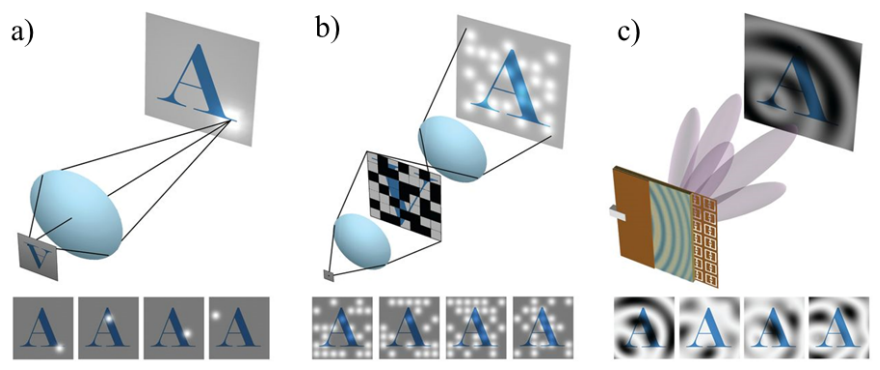

Fig. 1. a) Conventional imaging b) computational imaging c) computational imaging with a multiplexing antenna (from [28]).

wideband beams). Obviously, one could use a phased array to sculpt such patterns, but such an implementation carries the same cost and complexity of conventional techniques. A more pragmatic solution can be found by invoking metamaterial design concepts; employing metasurface antennas to generate spatially diverse patterns. They are simple, low-cost, require no power-hungry complicated hardware, and thus are a suitable candidate for implementing computational microwave imaging schemes and fulfilling the promise of low-cost and simple imaging devices.

This paper provides an in-depth review of the fusion of metamaterial design concepts and CI techniques to arrive at novel microwave imaging systems. We start by providing a more thorough background on these two groundbreaking ideas. Building upon these insights, we will describe a frequencydiverse metasurface antenna and the first realization of computational microwave imaging using metamaterial concepts. We will then review the evolution of frequency-diverse metasurfaces, and introduce a cavity feed mechanism to augment the abilities of such devices. Next, we describe a frequency-diverse imaging system capable of reconstructing images of humans at near video frame rate. We then introduce dynamic counterparts: metasurface antennas that generate electronicallytunable spatially diverse patterns. We illustrate their operation and expand upon the motivation behind their development. Different possibilities such as single-frequency imaging are illustrated. We conclude this paper with a vision for future microwave CI systems and outline several research directions.

\section{CO-DESIGN OF HARDWARE AND SOFTWARE OF MICROWAVE IMAGING}

\section{A. Computational imaging}

Computational imaging is an ever-growing topic at optical frequencies [17]. It is commonly described as the notion of intandem design of hardware and software of an imaging system to achieve desired imaging performance with improvement in hardware complexity, acquisition rate, novel capabilities, or a combination of them. In this framework, many materials and phenomena that were usually deemed undesirable for use in an imaging system are leveraged by including them in the CI scheme. A prominent example of such scenarios is the problem of imaging through scattering layers; the traditional methodology in this problem is to detect the direct light transferred/reflected through the medium (for example by time gating); in the CI framework, the effect of multiple scattering is included in the imaging formulation and accounted for, computationally, in image reconstruction [22]-[25], [29]. This insight resulted in the ability to image through paint or paper, or through biological tissues. In some cases, the multiple scattering of light was used to increase the resolution of imaging systems [30]. Another classical example where CI has revolutionized image formation hardware is the case of single pixel cameras [31]; in these systems, a single pixel detector, which is often much simpler to implement than an array, is used to image a scene of interest by multiplexing the scene information using a coded aperture or multiply scattering medium. In either case, by accounting for the impact of the multiplexing device in the image formation, non-conventional hardware that is simpler and/or faster may be used.

At microwave frequencies, the notion of CI has seen less exposure. The primary line of thought in this realm is to apply compressive algorithms [32]-[34]. In most of these works, the hardware is essentially a modification of traditional counterparts with a fewer number of antennas or lower sampling rates [35]-[38]. Rarely has a complete revamp of image hardware and software been examined until recently when frequency-diverse metasurface apertures demonstrated an alternative route toward microwave imaging.

\section{B. Metasurface antennas}

Metasurfaces have traditionally been viewed as surfaces decorated with periodically arranged metamaterial elements and with no or gradual variation, a condition that allows for application of averaging techniques (e.g. effective impedance or reactance) [39]. A plethora of works have been dedicated to designing metasurfaces that can shape the trans$\mathrm{mitted} / \mathrm{reflected} / \mathrm{refracted}$ waves in unprecedented manners, a prominent example of which is the Huygens metasurfaces [40]. At microwave frequencies, it is more convenient to combine the metasurfaces with a guided mode, giving rise to metasurface antennas where the metasurface is designed to interact with the guided wave to form a desired radiation pattern (or guide a surface wave). Generally speaking, metasurface antenna architectures share similarities with leaky wave antennas (LWAs) [41], and as a result, their design and operation have often been interpreted from the LWA perspective [42]-[44].

For metasurfaces in free space or used as antennas, the periodic condition on the variation of the metamaterial elements significantly simplifies the design process [45] but comes at an important cost; the range of possible operations available from the metasurface antenna are limited to those that are consistent with the slowly varying condition. Any deviation from this assumption can make the design of a metasurface using effective medium assumptions challenging. This condition also puts constraints on fabrication tolerances and requires electrically-large apertures (to avoid edge effects). Satisfying the slowly-varying requirement is also challenging for wideband applications. Inclusion of electronic tuning circuitry and altering the aperture, without violating periodic condition, can also be cumbersome. 


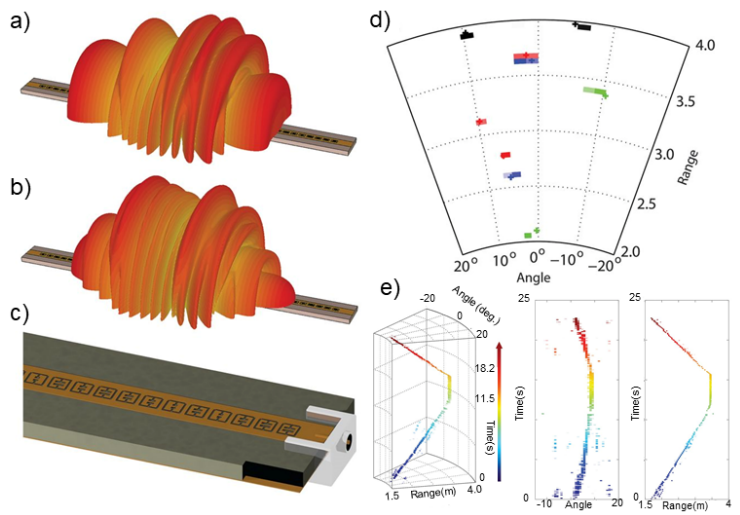

Fig. 2. a,b) Examples of frequency diverse radiation patterns (18.5 and 21.8 $\mathrm{GHz}$ respectively). c) Schematic of the frequency diverse metasurface. d) Reconstructed images of different static targets (denoted by different colors) and their respective locations. e) real-time imaging ( $10 \mathrm{~Hz}$ frame rate) of a moving object (from [28])

In the context of CI, metasurface antennas with periodically arranged identical (or gradually changing) inclusions have a more fundamental problem: the periodic uniform configuration limits the degrees of freedom, which is undesired in CI where we seek to generate as many diverse patterns as possible. Implementation of computational microwave imaging thus inspires a fundamentally different metasurface antenna; one with no inherent periodicity, where the properties of each element can vary significantly from neighboring ones. Such a chaotic arrangement, while not lending itself to averaging techniques, allows for the generation of multitude of spatially diverse patterns, and thus forms a useful hardware platform to implement CI schemes.

\section{First microwave CI using metasurface antennas}

The first realization of computational imaging with metasurface antennas strategically made use of a characteristic of metamaterial elements that has usually been deemed undesirable: narrow bandwidth. In this configuration, a microstrip excited an array of narrow bandwidth, resonant metamaterial elements whose resonance frequencies were selected randomly to lie within a broader frequency band of operation. As the guided wave propagates along the microstrip, it couples to the metamaterial elements and leaks to free space. The coupling to each element is determined by its resonance frequency. For each frequency, only a subset of elements radiates; as the frequency of excitation changes, different subsets are excited, giving rise to spatially diverse patterns as a function of frequency (referred to as frequency-diverse patterns). These patterns can encode the reflectivity map of a scene into a series of measurements indexed by frequency. The encoded signal is then post-processed to retrieve the image.

The microstrip imaging device, detailed in [28], is plotted in Fig. 2. It consists of a microstrip of length $40 \mathrm{~cm}$ with metamaterial elements embedded into its upper conductor. The resonance frequencies of these elements are randomly selected over the K-band (17.5-26.5 GHz). Specifically, complementary electric-inductive-capacitive elements (cELCs) [46], [47] are used as metamaterial radiators. To change their resonance frequencies, their geometrical size is changed (see Fig. 2 c)). Examples of the frequency diverse patterns produced by this antenna are also plotted in Fig. 2 a) and b).

To demonstrate imaging, the microstrip frequency diverse antenna was used to illuminate a scene of interest, with an open-ended waveguide (OEWG) collecting the return signal. Being inherently a one-dimensional aperture, the microstrip antenna could only image objects in two dimensions (range and one cross-range dimension). Assuming the first Born approximation and diffraction-limited imaging, the return signal $\mathrm{g}$ can be related to the reflectivity map of the scene, $\mathbf{f}$, through:

$$
\mathrm{g}=\mathbf{H f}
$$

where $\mathbf{H}$ is the sensing matrix (sometimes also referred to as the transfer matrix or the measurement matrix), that relates the return signal from the scene to the antenna radiation patterns through [48]:

$$
H_{i j}=\vec{E}_{i}^{T}\left(\overrightarrow{r_{j}}\right) \cdot \vec{E}_{i}^{R}\left(\overrightarrow{r_{j}}\right),
$$

where $\vec{E}_{i}^{T}\left(\overrightarrow{r_{j}}\right)$ is the field pattern radiated from the transmission aperture and $\vec{E}_{i}^{R}\left(\overrightarrow{r_{j}}\right)$ is the field radiated from the receive aperture field at the scene location $\vec{r}_{j}$. The index $i$ refers to the measurements (frequency points, in this case) while the index $j$ accounts for the voxels used to discretize the scene. As discussed earlier, the metasurface antenna at the center of this setup does not exhibit any apparent periodicity; hence, it cannot be modeled or characterized using averaging techniques. Instead, it was characterized experimentally using a near field scan [49]. The scanned data is propagated to the scene using simple plane wave decomposition techniques to compute the transmitted electric field.

Equation (1) can be solved to retrieve the scene reflectivity. In practice, $\mathbf{H}$ is usually not square and is typically illconditioned, and not generally subject to a straightforward matrix inversion. As a result, the reflectivity is retrieved by using computational techniques such as least square solvers or compressive reconstruction techniques [50]. An example of the imaging performance of this device is shown in Fig. 2 d). (for more, please refer to [28]). In this figure and in all others presented, the range direction is measured perpendicular to the metasurface, with the origin placed at the aperture surface. Since the data acquisition occurs over a simple frequency sweep at relatively fast speed, the microstrip metasurface antenna was able to demonstrate imaging of moving objects (see Fig. 2 e)). This proof-of-concept experiment provided early evidence of the possibility to revamp image formation at microwave frequencies; instead of antennas with well-defined directive beams that are switched or raster scanned, arbitrary and randomly selected frequency diverse patterns were used to obtain scene information. This demonstration, while modest in its imaging capabilities, laid the foundation for a new class of microwave imaging schemes.

\section{COMPUTATIONAL MICROWAVE IMAGING KEY FACTORS}

Before delving into the evolution of metasurface antenna designs for CI, we identify key factors influencing the imaging 
performance. As with all imaging systems, imaging performance is heavily dependent on the system resolution, which is ultimately determined by the aperture size and the frequency bandwidth [4], [51]-[54]. These parameters are well-known and are not examined here. We instead focus on the ones that are of particular interest in this framework.

Quality of measurements: The success of the reconstruction process in CI is proportional to the condition number of measurement matrix, $\mathbf{H}$. In the implementations examined here, it is generally the case that the number of measurements, $M$, is smaller than the number of unknowns (i.e. voxels) to be reconstructed, $N$, such that the sensing matrix is not square and hence does not have a direct inverse. Moreover, the radiated field patterns from metasurface antennas exhibit correlation, suggesting that the sensing matrix is not fully diagonal. Simply put, the higher the level of correlation among patterns, the lower the image quality. A primary goal in the following metasurface designs is thus to decrease the correlation of radiated patterns (see Fig. 8 of [55] for a qualitative comparison between the reconstructed images of a resolution target as a function of Q-factor).

Quantity of measurements: Another parameter of interest is the quantity of measurements possible from a system. Mathematically speaking, the maximum number of measurements is governed by the space-bandwidth product (SBP) [56]. In a practical implementation, the number of measurements is equal to the number of metamaterial radiators (due to the linear superposition of the field radiated by them). These notions have thus two important implications: we cannot improve imaging performance by increasing the number of elements beyond the SBP. Second, designing metasurfaces with larger numbers of radiators is of interest. This increase of elements (to increase the quantity of measurements) can, however, result in degradation of the measurement quality.

Signal fidelity: Because of their unusual and random radiation patterns, it is critical that metasurface antennas be characterized accurately. Near-field scanning of the antennas provides an excellent means to capture all complex phase variations of the illumination patterns [49]. Another factor vital to the signal fidelity is the alignment of the antennas, both during imaging [57] as well as during near-field scanning. As detailed in [57], the electrically-large size of the metasurface antennas make them susceptible to misalignment. Finally, precise calibration of the RF hardware is a necessary step, which can be accomplished by imaging known objects [58] or placing backscatterers in the scene [59].

Signal to Noise Ratio (SNR): Akin to any imaging system, SNR plays a key role in CI. However, its impact on the imaging performance is less crucial in computational schemes. This is due to the fact that the multiplexing nature of the illumination patterns used in this framework averages the effect of noise on reconstruction (sometimes referred to as Fellgett's or multiplex advantage [60], [61]).

Spatial sampling: In any imaging system, sampling the spatial content of the scene is vital to image quality. A uniform sampling of the spatial content results in a high quality image, while a sparse or nonuniform sampling can result in artifacts and aliasing in the reconstructed image. In the context of designing metasurface antennas for CI, this notion requires intelligent design of the placement of the metamaterial elements and their overall density across the aperture. In other words, while the number of elements are selected by balancing a trade-off between measurement quantity and quality, their distribution has to also be designed to match requirements for a uniform sampling of the spatial frequencies.

To better illustrate the intricate interplay of these factors, let us consider an alternative architecture to the frequencydiverse metasurfaces of Section II-C: a LWA or a Fabry-Perot resonant cavity. The former can generate a frequency-steered narrow beam interrogating a scene, while the latter can probe the scene using a pencil beam at broadside to a conical beam as the frequency changes. Both structures can form an almost diagonal sensing matrix and retrieve information in the cross range; however, they both under-sample the scene: each angle is probed by a single frequency and, as a result, reconstructed images suffer from aliasing. A similar idea has been pursued in [62] in a reflective configuration where two gratings are used to image a scene of interest-gratings support modes in a similar fashion as the leaky-modes of a LWA or resonant Fabry-Perot. While the gratings can map the cross-range information at each frequency to certain points in the scene, they are not suitable for imaging 3D objects. One remedy is to use Doppler shift, which allows such systems to track small targets, but still cannot result in 3D images of a complex scene.

\section{FREQUENCY-DIVERSE METASURFACE ANTENNAS}

\section{A. Frequency diversity via element's resonance}

The first frequency-diverse metasurface implementation discussed earlier used diversity of metamaterial elements' resonance frequencies [28]. This idea was later extended to 2D apertures, where a parallel plate waveguide replaced the microstrip line [63]-[65]. In this framework, realizing frequency diversity depends on the sharp resonance of the metamaterial radiators. The sharpness of the element resonance can be quantified by its quality factor (Q-factor): Increasing the Qfactor of the constituent metamaterial element is an effective tool to reduce the correlation between the illumination patterns produced by the metasurface antenna. However, in a practical implementation, the Q-factor cannot be increased infinitely; the strong resonance of the element also results in high dielectric and conduction losses, limiting the practical Q-factor. For small antennas, the Q-factor is by definition inversely proportional to the equivalent resistance [66]. The resistance of the antenna is usually a combination of radiative and ohmic losses. In our case, where the metamaterial element is a complementary resonant structure embedded into a waveguide, the high electric current and/or fields associated with the resonant nature of the element usually prevent the element from exhibiting low losses. As a result, increasing the Q-factor of the resonant, metamaterial elements is generally accompanied by a decrease in radiation resistance, and as a result, lower radiated power and radiation efficiency. Therefore, a careful optimization procedure is needed to find an optimum Q-factor/radiation efficiency ratio for successful imaging. 

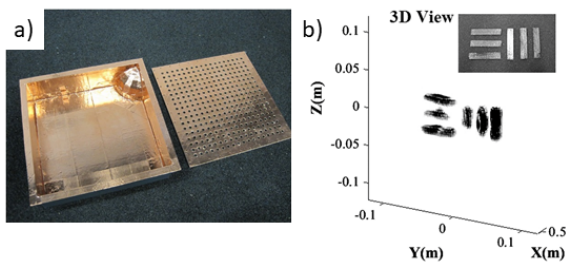

Fig. 3. a) A disordered cavity with one side perforated with an array of irises (transmittive metasurface layer). b) Example reconstructed image (from [67]).

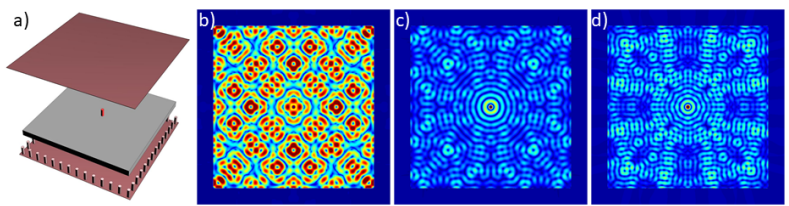

Fig. 4. a) An electrically-large printed cavity $\left(10 \times 10 \times 0.15 \mathrm{~cm}^{3}\right)$. Simulated magnetic field inside the cavity at example frequencies: b) $17.5 \mathrm{GHz}$ c) 22 $\mathrm{GHz}$ d) $26.5 \mathrm{GHz}$ (from [71]).

\section{B. Frequency diversity via feed diversity}

To alleviate the stringent trade-off governing Q-factor and efficiency of a metamaterial element, an alternative approach is to utilize a different mechanism to realize frequency diversity; essentially separate the factors responsible for frequency diversity and radiation. Toward this goal, [67] proposed using spatially-diverse guided-modes within the feeding structure to excite the metamaterial elements. A significant advantage of this technique is that sampling the guided-mode can be done using weakly-resonant meta-elements exhibiting minimal dielectric and conduction losses, without stringent constraints on the radiation efficiency and Q-factor. The frequency-diverse guided wave in [67] was realized utilizing a disordered electrically large cavity $\left(20 \times 20 \times 5.3 \mathrm{~cm}^{3}\right.$ with a spherical deformation having a radius of $4.5 \mathrm{~cm}$ ), inspired by a counterpart in ultrasound [68]. Such a feed mechanism, shown in Fig. 3 a), supports many modes at K-band. As the frequency of operation changes, different modes are excited, which are projected by the metasurface layer-acting as a partially transmissive layer-onto the imaging domain.

With the excellent imaging performance demonstrated using cavity backed metasurface (see Fig. 3b), this idea was later adapted to planar 2D configurations by employing a printed cavity, as shown in Fig. 4 [69]-[71]. This device consists of a metasurface printed on a grounded dielectric substrate. The edges of the substrate are terminated in conducting walls, forming a printed cavity with heights less than a halfwavelength thick, thus supporting a single mode excitation along its broadside direction. The simulated field distribution inside the printed cavity is shown in Fig. 4. Analyzing the cavity mode at three different frequencies, $17.5 \mathrm{GHz}, 22 \mathrm{GHz}$ and $26.5 \mathrm{GHz}$, it is evident that the mode pattern can be engineered to be extremely diverse.

Using electrically large cavities with their well-established high Q-factors can thus significantly boost the frequency diversity. However, the actual factor affecting the performance is the loaded Q-factor which includes losses due to radiation
TABLE I

VARIATION OF THE LOADED Q-FACTOR AND THE RADIATION EFFICIENCY AS THE NUMBER OF RADIATORS INCREASES (FROM [69])

\begin{tabular}{c|c|c|} 
Num. of radiators & Q-factor & Radiation Efficiency \\
\hline \hline 0 & 254 & $0 \%$ \\
4 & 225 & $6.2 \%$ \\
16 & 184 & $26.2 \%$ \\
64 & 123 & $62.1 \%$ \\
\hline \hline
\end{tabular}

from the metasurface layer. This notion is demonstrated using experimental characterization of printed metasurface-cavities in Table. I [69]. The variation of the loaded Q-factor as a function of radiative layer means we still need to balance a trade-off between the radiation losses and the loaded Q-factor. To better understand the intricacies of this trade-off, recall from the discussion in Section III that the total number of distinct illumination patterns possible is equal to the number of metamaterial radiators. This means we should increase the number of elements as much as we can. However, that would increase the radiation loss, and in return, reduce the loaded Qfactor (and thus the quality of the measurements). The optimal number of metamaterial radiators should also be selected by ensuring a dense and uniform sampling of spatial frequency domain (k-vectors).

The theoretical investigation in [72] laid the foundation for determining the useful number of elements given a bandwidth and Q-factor. In this work, it is shown the number of elements needs to be approximately equal to $Q B W / f_{c}$, where $B W$ is the bandwdith of operation and $f_{c}$ is the center frequency. To increase number of measurements without sacrificing Q-factor too much, it was also suggested to use a cavity-metasurface as the receiver [72]. In this case, the number of radiators on the transmitter $N_{T X}$ and on the receiver $N_{R X}$ should satisfy:

$$
Q=\frac{N_{T X} N_{R X} f_{c}}{B W}
$$

Next, the layout of these radiating elements needs to be designed. Recalling discussion of Section III, the thinned elements need to be placed intelligently to avoid aliasing. Toward this goal, [73] used the effective aperture principle to determined ideal population density of the thinned metasurface aperture. Fig. 5 provides the results of this analysis for a pair of transmit and receive metasurface antennas with their meta-elements a) populating the entire antenna aperture and arranged in a Fibonacci pattern and b) arranged in a MillsCross configuration. The overall effective aperture patterns of the metasurface antennas are also depicted. To calculate the effective aperture patterns, the aperture field distributions of the transmit and receive metasurface antennas are convolved and summed over the K-band frequencies.

A careful investigation of Fig. 5 reveals that whereas both effective apertures have the same k-space extent in the spectral frequency domain, the resultant effective aperture pattern for the Mills-Cross configuration exhibits significantly less redundant sampling combinations. More importantly, this uniform sampling is accomplished using fewer radiating elements.

The frequency-diverse metasurface of Fig. 5 was later further optimized where irises were replaced by elongated 

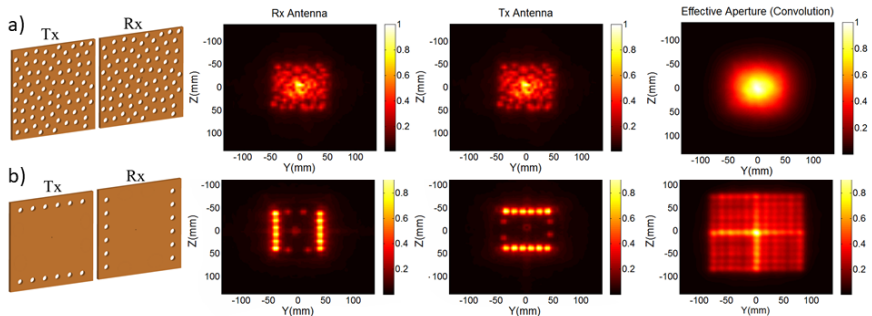

Fig. 5. Different configurations of transmit and receiver apertures (each $10 \mathrm{~cm} \times 10 \mathrm{~cm}$ ) and their corresponding effective aperture. a) dense array populating the whole aperture b) Mills cross configuration (from [73]).

slots and the cavity was deformed to further reverberate the guided wave. The result, shown in Fig. 6, had a measured Qfactor of 330. According to (3), the upper bound number of distinct measurements is 135 . By using $N_{T x}=N_{R x}=16$, a compromise between maximizing spatial frequency sampling and maintaining the necessary SNR was achieved. Furthermore, K-band was sampled at 101 frequency points, satisfying the upper-bound limit on the number of distinct measurement modes. Using more than 135 frequency points would bring highly redundant information. This aperture is used in the next subsection as the basis for imaging humans.

\section{High frame-rate microwave imaging of humans}

The ability of the frequency-diverse metasurfaces to retrieve scene information in fast frequency sweeps promises fast security screening systems. This proposal was verified in [74] where a multi-static system consisting of 24 transmitting and 72 receiving cavity-backed metasurface antennas (each 12 $\mathrm{cm} \times 12 \mathrm{~cm}$ ) was built (see Fig. 6). The number of transmitters and receivers, as well as their layout, were designed based on the earlier discussion on quality and quantity of measurements. However, this optimization was conducted within the strict restriction imposed by the RF hardware on the modular format of the assembled system where each module could only support 2 transmitter and 6 receivers. Using 12 modules, the overall aperture size was $2.1 \mathrm{~m} \times 2.1 \mathrm{~m}$ (see Fig. 6).

A depiction of the spatially diverse field patterns interrogating the scene containing a human is given in Fig. 6. Representative images are shown in Fig. 7 where high-fidelity reconstructions of a human-sized mannequin placed in different locations within the scene are demonstrated. The highquality reconstructions validate the proposal that frequencydiverse metasurface antennas can be used to develop novel security screening systems; they also show a significant advantage in that the system can image the target in any location within the region of interest. As discussed in [74], [75], these images show similar quality to those that would be possible with conventional techniques, but at much lower cost and complexity level. The simplification can be better appreciated by comparing the 96 antennas used in this system to the number of antennas required to synthesize the same aperture at the Nyquist limit using simple dipole antennas; i.e. 124,000 antennas. Interestingly, the proposed system can also image multiple humans at once. More importantly, this system is capable of retrieving images of humans at $7 \mathrm{~Hz}$ (with the
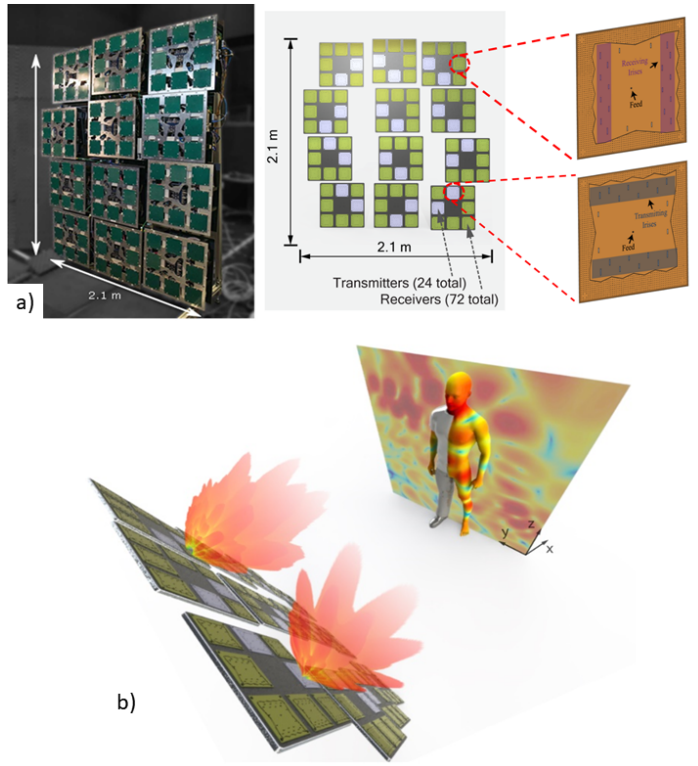

Fig. 6. a) General configuration of a security screening system based on frequency-diverse metasurface antennas. b) Multiplexing of the reflectivity map of human targets by the frequency-diverse patterns (from [74]).

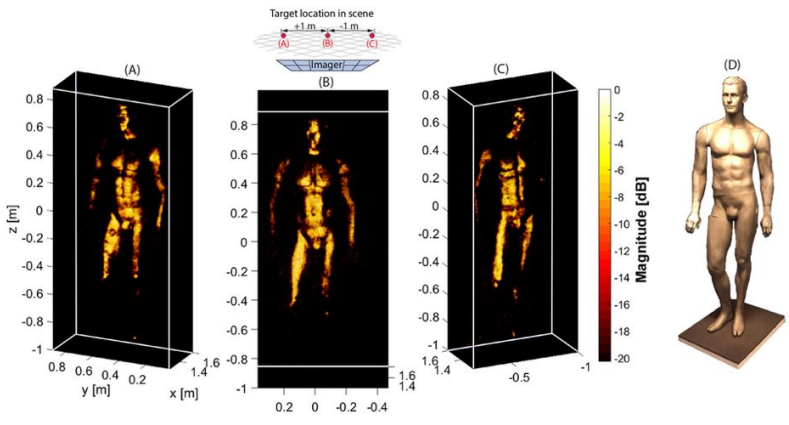

Fig. 7. Reconstructed image of a human-sized mannequin using the system of Fig. 6 (from [74]).

potential to be increased to $100 \mathrm{HZ}$ ) [76], [77], a remarkably high acquisition-rate at microwave frequencies. It is worth noting that the above mentioned rate only considers data acquisition; reconstruction of images may take longer. Some recent works have tackled the slow reconstruction problem and demonstrated the potential to achieve $4 \mathrm{~Hz}$ reconstruction speed (see e.g. [78], [79]):

\section{DyNAMiC METASURface ANTENNAS}

The remarkable performance of the frequency-diverse imaging system points toward a paradigm shift in microwave imaging, in particular for close-range applications (e.g. for security screening). However, using frequency-diverse apertures mandates employing ultrawide bandwidths, a trait that can be limiting in many applications. Ultrawideband operation drives up the cost and complexity of the RF hardware. It can also be prohibitively expensive or impossible to employ given the overly congested microwave spectrum. While certain security applications can accept these economic constraints, costs can become the bottleneck in many other applications. Furthermore, the quantity and quality of illumination patterns possible 


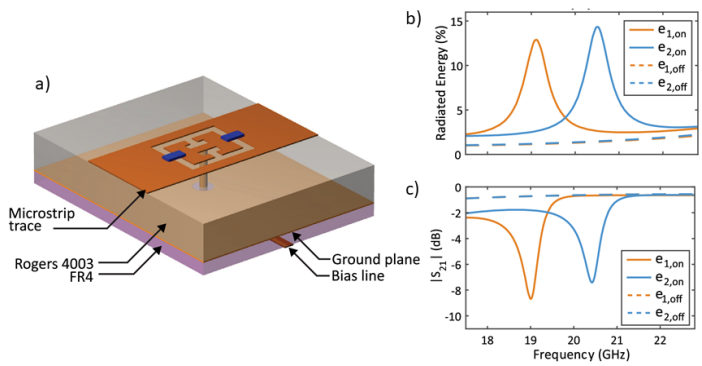

Fig. 8. a) configuration of a waveguide-fed tunable metamaterial element. b) Simulated insertion loss. c) Simulated radiated power (from [80]).

through the means of frequency-diverse apertures are closely tied to a stringent trade-off between Q-factor, the quality and quantity of measurements, and SNR, as discussed earlier. Adding bandwidth limitations imposed by spectrum allotment or interference regulations to these trade-offs can significantly deplete the design space for frequency-diverse apertures. These drawbacks inspired efforts to replace frequency diversity with electronic reconfiguration. A conventional approach is to use active or passive phased array or switch arrays. A more pragmatic approach has been proposed in the form of dynamic metasurface antennas (DMAs), which is the subject of this section.

\section{A. General configuration of DMAs}

The DMA shares the same architecture as the frequencydiverse aperture in that it consists of a waveguide or a cavity exciting an array of metamaterial radiators. In contrast to the frequency diverse case, each element is loaded with a switchable component such as a PIN diode that is addressable independently. In this framework, by tuning the response of different subsets of the metamaterial radiators, the illumination pattern can be changed even at a single frequency. There are a variety of options for the tunable component, each with its own pros and cons. Here we focus on PIN diodes as the tuning mechanism, which can render an element radiating on or nonradiating off (sometimes referred to as binary tuning). When the element is non-radiating, most of the energy incident on it passes through to the next element, while when it is radiating, a portion of the energy is leaked as radiation (see Fig. 8). PIN diodes however provide a limited degree of freedom and are also current driven (i.e. consume more power). Another option is to use varactor diodes, which can shift the resonant frequency of an element by application of different levels of DC voltage (thus referred to as grayscale tuning). They essentially provide control over both amplitude and phase of the metamaterial element (though not independently). Thus, varactors provide more degrees of freedom compared to PIN diodes. Furthermore, they are voltage-driven and consume much less power. On the other hand, varactors need a more complicated tuning circuitry that allows for gradual change in the DC voltage, and are also prone to exhibit nonlinear response. Moreover, since they cannot turn an element completely off, they can lead to fast depletion of the guided wave.

An important distinction of the DMAs examined here and the ones studied in previous works, i.e. electronically reconfig-
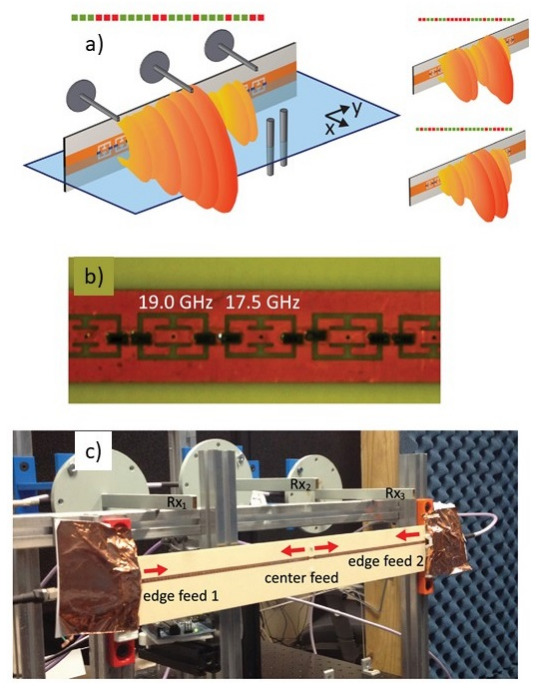

Fig. 9. a) Sample radiation patterns and the corresponding tuning states (green and red denote on and off, respectively). b) close-up view of the fabricated sample. c) Imaging system consisting of the 1D DMA (from [80]).

urable antennas or metasurfaces [81]-[87], lies in the fact that each element is individually addressed here and independent of the voltage applied to other elements (analogous to a spatial light modulator at optical frequencies). In the large body of work dedicated to reconfigurable metasurface antennas, tuning is used to change the effective impedance or reactance of a metasurface aperture. While implementation of such a tuning circuitry is easier, it does not provide sufficient freedom to arbitrarily change the radiation pattern (i.e. as would be possible if the response of all elements are tuned independently). In contrast, here we change each resonance response of each element individually. This option, while offering more degrees of freedom, requires a more involved design to bring DC tuning circuitry to each element. As detailed in [88], two main modifications to the metamaterial element are needed: a metallic via that goes through the ground plane (through a small hole) and connects to each metamaterial element, and a radial stub on the other side of the via to decouple RF and DC signals. This configuration is shown in Fig. 8. It is worth noting that some reconfigurable antennas mentioned before [83], [85], also tune each impedance element of a modulated impedance antenna individually. However, the voltage applied to each element is not independent of other ones; they need to maintain the modulated impedance condition to allow for the structure to support leaky/surface modes. In contrast, here we turn each element on or off independent of other elements.

\section{B. Evolution of DMAs for computational imaging}

The first DMA designed for CI was reported in [89]. It consisted of eight metamaterial elements embedded into the upper conductor of a microstrip line. Each element was loaded with two PIN diodes. This device successfully generated spatially diverse patterns that could be altered by turning different subsets of PIN diodes on or off. Given the limited number of elements and the small aperture size, the imaging performance 

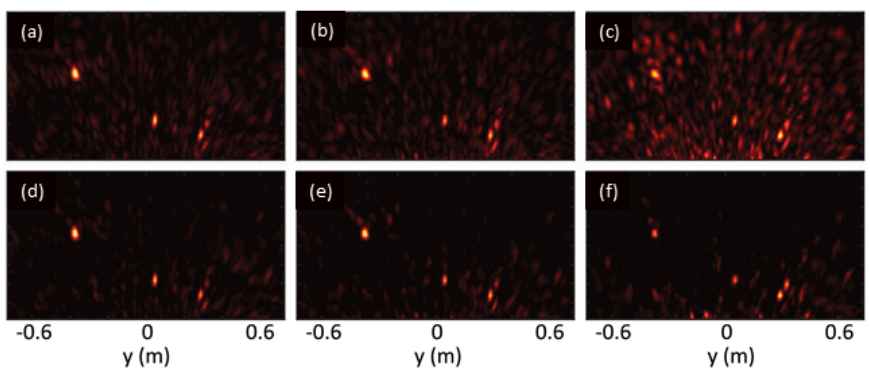

Fig. 10. Top down view of the reconstructed images of three metallic cylinder at different locations using 1D DMA. a)-c) uses GMRES and d)-f) uses Twist. The number of masks utilized are 26, 15, and 7 masks from left to right (compression ratios of roughly $1: 1,1: 1.75,1: 4)$ (from [80]).

of this DMA was limited, but validated the promise of DMAs for CI.

The second DMA was designed to include more elements [80]. It consisted of a microstrip fed from the edges as well as the center. The elements were designed to have two different resonance frequencies (17.5 and $19 \mathrm{GHz}$ ) interleaved along the aperture (see Fig. 9). This configuration broke symmetry and pushed the Bragg reflection out of the band of operation, while ensuring the aperture could operate over a desired band. Each element was loaded with two PIN diodes and addressed independently. The diodes were placed at the gap of the metamaterial element with high concentration of electric field. The spacing of the elements was subwavelength (approximately a fifth of a wavelength). This choice was due to the fact that binary tuning alone does not offer sufficient modulation freedom. By placing elements densely, we can utilize the phase accumulation by the guided wave exciting elements to ensure diverse phase and amplitude among radiating elements. It is worth emphasizing that the number of distinct measurements possible is still determined by the SBP and is less than the number of elements.

The size of the aperture was selected such that it guaranteed ample power reached each element. As the guided wave travels along the microstrip and couples to the elements, it loses its power. In addition, an off element also contributes to the decay due to ohmic losses of the PIN diodes. Considering these two factors from full wave simulations, and the fact that the microstrip is fed from 3 locations, the length of the DMA was selected to be $40 \mathrm{~cm}$ with 112 elements.

The fabricated sample is shown in Fig. 9. Near field scans were used to experimentally characterize this DMA for different tuning states and frequencies. Sample radiation patterns are also shown in Fig. 9. Spatially diverse patterns that are altered as a function of the tuning states are evident. These patterns correspond to tuning states with on elements randomly selected. The random distribution of on elements mitigates aliasing. The number of on elements are selected by balancing two factors: spatial distinction between the generated patterns and SNR. To ensure the former, the trivial solution is to use tuning states with a single element on at a time. While guaranteeing orthogonality, this choice leads to low radiated power, significantly degrading the SNR. It also does not allow for multiplexing of the information. The other extreme where

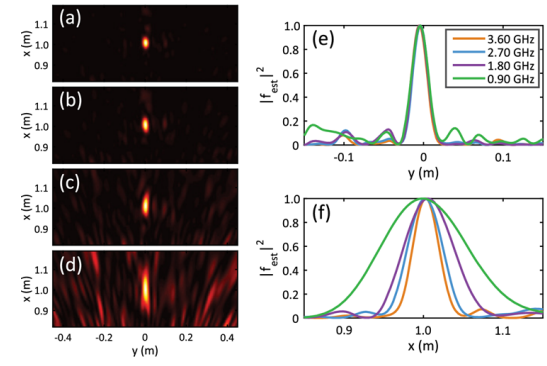

Fig. 11. Reconstructed images as the bandwidth is reduced a)-d) are top down view, while e) is the cross range and f) is the range plots. (from [80])

only few elements are off is also not practical: while the radiated power is high in this case, the guided wave depletes rapidly before reaching most of the elements, rendering many of the patterns similar. Taking into account the feed mechanism and the losses due to radiation and PIN diodes, masks with almost a quarter of the elements radiating were used for most of the studies.

The imaging performance of this device, utilized in the setup shown in Fig. 9, is plotted in Fig. 10. Here, the DMA acts as a transmitter illuminating a scene of interest, while three OEWGs collect the scattered signal. The image reconstruction is similar to the frequency diverse case, with the modification that the measurement index now also loops through the tuning states. Sample images shown in Fig. 10 clearly show different objects resolved in both range and cross range. More importantly, it demonstrates the ability of the 1D DMA to reduce the required number of measurements (increase acquisition rate). To visualize the fact that this device resolves objects in the cross range by multiplexing information via tuning masks (and not frequency diversity or bandwidth), reconstructed images of an object in range and cross range as we decrease the bandwidth are shown in Fig. 11. By reducing the bandwidth, we are still able to resolve the object in the cross range. This result clearly evidences the utility of a DMA to multiplex information and its promise as the central hardware of a microwave CI system.

\section{Adaptation of range migration algorithm (RMA) and de- velopment of a hybrid imaging system}

The images presented in the previous subsection have been obtained through inversion of the forward model (see (1)) by matched filter, least squares techniques, or compressive algorithms. While this approach is effective and straightforward, imaging large scenes using this method requires memory intensive storage of the field values comprising the sensing matrix, $\mathbf{H}$, and the necessary manipulations of this matrix can quickly become computationally prohibitive. Alternatively, Fourier domain reconstruction methods have historically enabled drastic computational savings due to the efficiency of the Fast Fourier Transform (FFT) [52], [90], [91]. However, such techniques have largely been applied to antenna arrays with well-defined phase centers and uniform spacing. At first sight, it may seem that the DMA imaging scheme may not be compatible with such techniques. However, as detailed in [92], the data set collected by the DMA imaging system 

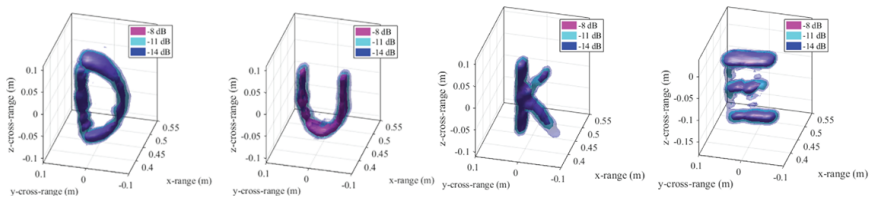

Fig. 12. Volumetric images reconstructed by a hybrid DMA-SAR system using modified RMA (from [93]).

can be transformed to one compatible with Fourier transform reconstruction techniques. A more interested reader can refer to [92], [93] for the detailed mathematical derivations. Here, we only describe the physical intuition behind this transformation: for each particular tuning state of a DMA, the corresponding pattern can be described as the superposition of the scattering response of the collection of metamaterial elements (some on and some off). In other words, we can envision the spatially diverse patterns to be due to a virtual array of dipoles replacing the DMA. Each tuning state then corresponds to this array of dipoles radiating with complex amplitude that is acquired during near field scan (by invoking surface equivalent principle). This insight can thus be used to replace the radiation pattern of the DMA with that of a uniform virtual dipole array, allowing for application of standard array imaging principles such as RMA.

Adaptation of RMA (commonly used in SAR systems) to imaging with DMAs opens up a unique opportunity: a hybrid system that utilizes a DMA efficiently in a SAR configuration. This is especially attractive for the 1D DMA which can only image objects in 2D, i.e. the plane containing the DMA. To extend these results to 3D (volumetric imaging) a trivial solution is to mechanically move the DMA in the other direction to retrieve images in that direction. This hybrid imaging system has been detailed in [93] and is not repeated here. As an example, images obtained by moving the DMA in both directions are presented in Fig 12. We clearly see that this hybrid system can obtain images of high quality. What is more remarkable is the speed up possible by using modified RMA; given the particularly large scene sizes interrogated in this hybrid DMA-SAR system, the adapted RMA offers significant computational advantages over matrix inversion methods (on the order of 200 times). Put differently, while DMAs alleviate the hardware constraints of large imaging systems, the adapted RMA technique reduces software demands in terms of memory and computational complexity, enabling a system that is simple with respect to both hardware and software.

\section{Single frequency imaging}

The results of [80] suggest the possibility to retrieve cross range information even at a single frequency by reconfiguring the DMA's illumination patterns. This demonstration raised another intriguing question: is it possible to retrieve both range and cross range information at a single frequency? At first glance, this idea may seem counter intuitive and opposite to the general understanding that bandwidth dictates range resolution [53]. However, the linear relationship between range resolution and bandwidth, widely quoted in the radar and microwave

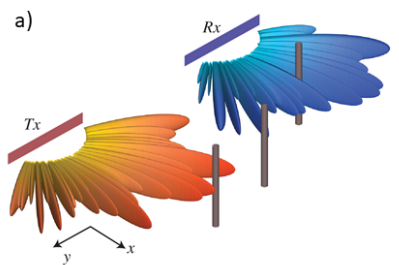

b)
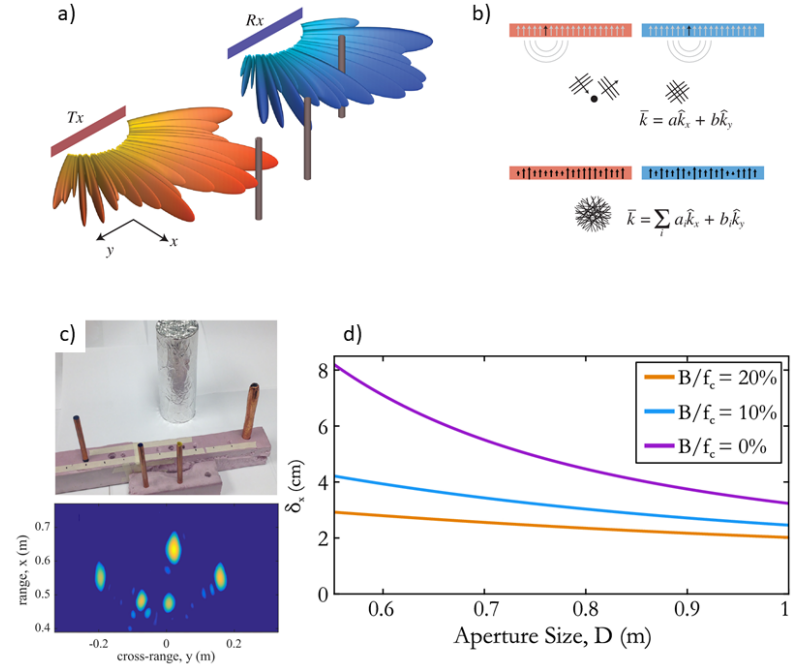

Fig. 13. a) Schematic of single-frequency imaging using 2 DMAs. b) Multiplexing of different look angles using DMAs. c) Reconstructed images at a single frequency. d) Dependency of range resolution on bandwidth and the aperture size (from [95]).

imaging literature [53], is only applicable in the far field. Close to an imaging apparatus, where the imaging device acquires many looks of a target, including those at oblique angles, the objects along the range direction can also be resolved, even at a single frequency. This proposal may be better explained by examining the k-vector coverage of an imaging system, similar to that used in [94], operating in the near field and retrieving both range and cross range at a single frequency. In this setup, a transmitter and a receiver are placed on translational stages side by side. For each transmit location, the receiver sampled the signal along the entire receiving aperture. When the transmitter and receiver are at oblique angles from the scene, the respective k-vectors have components both along the range and cross range. This notion thus translated in the ability to resolve objects in both directions at a single frequency.

The system used in [94] was extremely slow and bulky. A more pragmatic solution can be envisioned by multiplexing the $\mathrm{k}$ vectors in one measurement (instead of one at a time). Such a capability is readily available from the DMA as documented earlier. The setup to implement single frequency imaging based on this idea is shown in Fig. 13 where each of the moving transmitters and receivers are replaced by one DMA [95]. The corresponding analogy between this setup and the translated antennas of [94], and the multiplexing of different $\mathrm{k}$-vectors is visualized in Fig. 13. This proposal was also verified by the images shown in Fig. 13. We clearly see objects that have been resolved in both range and cross range at a single frequency. The dependency of the range resolution on the overall aperture size as the bandwidth is reduced is also depicted in Fig. 13. The concept of single frequency imaging has also been extended to 3D scenarios by moving the DMAs in the direction normal to their orientation (see [96]).

While it may seem operating at close proximity to the imaging aperture is a limiting factor, a variety of applications in fact involve microwave imaging at close range (e.g. security screening and through wall imaging) and can benefit from 

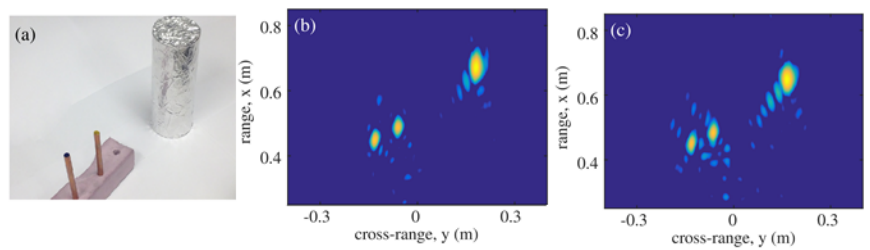

Fig. 14. a) Scene of interest. b) reconstructed images with no misalignment and c) with $2.54 \mathrm{~cm}$ misalignment in the $\mathrm{x}$ and $\mathrm{y}$ direction (from [95]).

single frequency operation which brings unique opportunities and advantages. Two obvious advantages of single frequency operation are the simplification of the RF hardware and the alleviation of concerns about spectrum allotment and interference. A more practical advantage is the fact that single frequency measurement does not require accurate phase measurement (still requires stable phase measurement). Any phase error is the same for all the measurements and essentially translates into a simple phase factor multiplying all measurements and does not affect qualitative images of reflectivity maps. This robustness to phase error relaxes constraints on calibration and alignment as documented in [95] and plotted in Fig. 14-traits that can be extremely useful in practical implementations [57]. Another way to interpret this robustness is that the single frequency imaging system is not perturbed by thin dielectric layers (e.g. walls) in front of it. They only cause a constant phase error across all measurements. Note that the phase error caused by the wall's unknown material is still angle dependent, and can cause degradation of imaging even at a single frequency if simple-pattern antennas are used. However, every DMA measurement consists of many look angles, each angle with different phase error, that on average, result in a constant phase error across all measurements. This means a single frequency DMA-based system can image through dielectric layers without requiring knowledge of the thickness or material, as experimentally verified in [].

\section{E. Phaseless (Ghost) imaging}

As discussed above, DMAs can operate at a single frequency and offer imaging capabilities with drastic hardware complexity and cost reductions. Whereas the implementations described above, as well as most other microwave imaging systems, utilize both the phase and amplitude of the scattered fields for reconstruction, further system simplification can be achieved through phaseless imaging methods. Acquisition of intensity-only microwave data demands no phase synchronization between transmitting and receiving elements [97], [98], and is generally less sensitive to phase error and phase drift over long integration times [99]. In [97], phaseless imaging was demonstrated using frequency-diverse apertures by applying a phase retrieval algorithm to the intensity-only dataset. In contrast, DMAs pave the way for forming images from intensity measurements by leveraging their ability to generate a collection of spatially diverse illumination patterns [100]. This imaging scheme can be interpreted in the language of the statistical correlation technique termed ghost imaging
[27], and was recently demonstrated at microwave frequencies using the above mentioned DMAs in [100], [101].

\section{DISCUSSION AND FUTURE WORK}

Previous sections have compiled an in-depth overview of metasurface antennas and their fusion with computational techniques to enable radically different imaging schemes. These accomplishments in our opinion represent just the beginning of many other novel different imaging and sensing ideas yet to be discovered. As the inception of metamaterial concepts spurred a revisit of many mature technologies and triggered new discoveries, the fusion of metamaterial design concepts and CI techniques are also poised to spur exploration of many new directions. In this section, we review some of these new ideas and propose some other future directions.

\section{A. Multi-look human imaging}

The fast frame rate possible with the metasurface imaging systems opens up unique opportunities. For example, multiple looks of the same target as they walk across a scene can be stitched together to form a complete image of the person without requiring time consuming stop and pose process of conventional systems [74]. Applying classification algorithms to the stitched image or each frame is another future direction. Another opportunity is to add on-demand super-resolution to the imaging process via hardware or software modifications. The former can be realized by using DMAs that can form a focused image (spotlight) over an area of interest to retrieve higher resolution images [102]. The later can be accomplished by applying recent ideas from image processing techniques on subwavelength imaging techniques (e.g. [103], [104]).

\section{B. Far-field imaging}

Most systems examined up to now conduct imaging within few meters. This limit is driven by two factors: 1) many applications such as security screening and through-wallimaging are usually conducted at distances within few meters. 2) The ambiguous range, set by the frequency sampling, limits the range of imaging. For example, in the security screening configuration of Fig. 6, imaging is conducted with $90 \mathrm{MHz}$ frequency steps, which translate into $1.66 \mathrm{~m}$ ambiguous range; if we image beyond that, we start to see replicas of the same image. Note that this is true for any imaging system regardless of using metasurfaces or computational techniques.

For imaging farther away, use of directive pencil beams are more common. DMAs are also uniquely well-suited for such applications due to their lightweight, low-cost, and planar platform for electronically steered antennas. Whereas the DMA implementations discussed here targeted radiation patterns suitable for CI, generating directive beams and steering them using a similar configuration is also a possibility. In the beamforming framework, the array of the reconfigurable metamaterial radiators acts similar to a hologram that can be designed to form a prescribed directive beam. The design and operation of DMAs in this context are investigated in other works and are not repeated here [13], [105], [106]. 


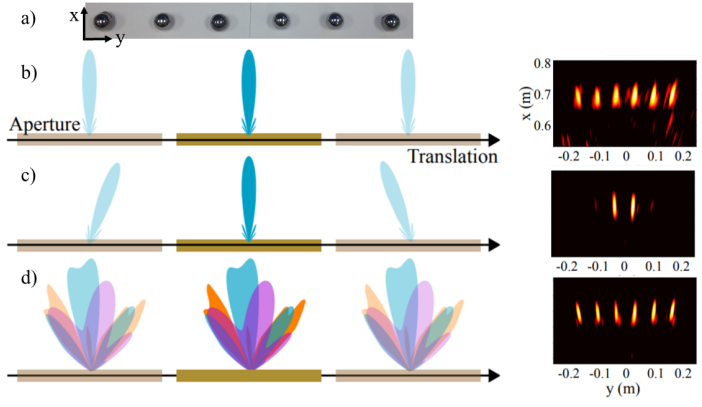

Fig. 15. a) Target to be imaged using a single DMA in b) stripmap SAR c) Spotlight SAR d) Diverse-pattern SAR modalities (from [112]).

However, it is worth emphasizing two important advantages that DMAs can bring to far field imaging: their promised lowcost and simple hardware can remove economical obstacles to implement multi-static MIMO imaging systems [11]. Within such an imaging scheme, various innovative imaging modalities can be envisaged (e.g. spotlight, polarimetric, coincidence imaging, etc.) [107]-[110]. Second is the flexibility of the metasurface antenna architecture. Several or all of imaging capabilities can be realized using the same hardware. In fact, DMAs' reconfigurable nature can be leveraged to develop an adaptive imaging scenario: an imaging system that can change its imaging modality based on the demand of the operation and features of the target under investigation. An example of this dexterity is shown in Fig. 15. We can see that depending on the application, the DMA can realize wide field of view imaging (stripmap), high resolution imaging (spotlight), or somewhere in between the two (diverse pattern SAR) [107], [111], [112].

For far-field imaging, one may also consider LWAs. However, the primary challenge with LWAs is their frequency squinting, which may result in an undersampled k-space coverage. If, by introducing reconfigurable components, one can maintain patterns at all frequencies, LWAs can also be considered for such applications [83], [85]. In that case, both configurations are viable options and depending on specific application and constraints, one may use either of them.

\section{C. $2 D$ DMAs}

Extending the concept of DMAs to two dimensions is another opportunity going forward. An example of such a configuration was demonstrated in [113]. An interesting feature of this device was that different mechanisms were used for radiation and reconfiguring the generated patterns. In the DMAs examined before [80], the tunable component is integrated into the resonant radiating element. In [113], a cavity similar to that of [67] was used, with the exception that one of its bounding walls contained a reconfigurable impedance surface [114] (see Fig. 16). By changing the boundary condition inside the cavity, the electromagnetic field formed within the cavity was changed, and as a result, the field projected into the scene of interest was also modulated. This device provides an excellent platform to compare the DMA's performance with that of its frequency-diverse counterpart (see imaging results of Fig. 16). However, the tunable cavity imager

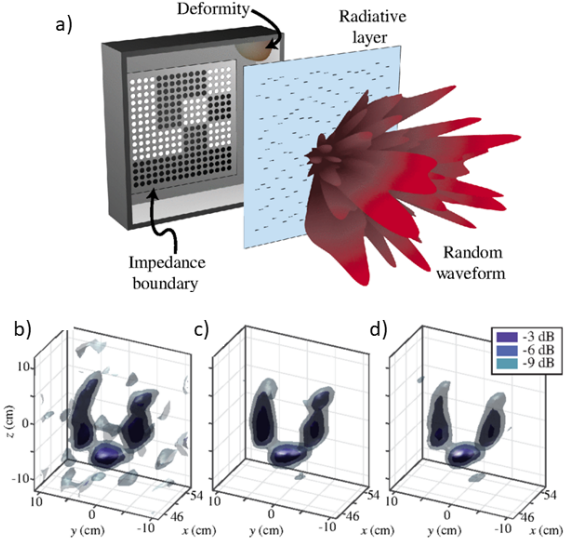

Fig. 16. a) Schematic of a tunable cavity $\left(11 \times 11 \times 5.5 \mathrm{~cm}^{3}\right)$. Reconstructed images with b) 201 frequency points and single mask c) 26 frequency points and 8 masks. b) 26 frequency points and 4 masks (from [113]).

was bulky and unfavorable for practical application. A planar version of a 2D DMA has been envisioned in [115]. In this configuration, a planar cavity similar to the ones introduced earlier is used. In contrast, each element is loaded with a tunable component. The numerical results presented in [115] suggest that such a device can alleviate many trade-offs of the frequency diverse system. It also demonstrated a static volumetric single frequency imaging. This device has already been fabricated and its imaging performance is reported in [].

An alternative option is to use an array of 1D DMAs to form a 2D aperture [7], [116]. However, to feed such structures with one port requires a reconfigurable corporate feed to ensure uniform aperture field distribution, significantly increasing the complexity of this device. The 2D DMAs proposed in [113], [115] offer much simpler configuration to generate spatially diverse patterns from a single port. Nevertheless, the array of 1D DMAs may offer exciting opportunity for generating electronically reconfigurable directive beams [116].

\section{Modeling and potentials for optimization}

As noted earlier, the metamaterial antenna architecture used in the context of $\mathrm{CI}$ cannot be modeled using averaging techniques due to drastic variation of constituting elements. A brute-force solution is to utilize full wave solvers. However, the computational needs for such electrically large structures with subwavelength variations can easily become intractable. To address this issue, initial works used simplifying approximations to model these metasurface antennas as array of dipoles whose complex magnitude was determined by the guided wave [48], [64], [71], [115]. While many factors, such as radiative losses or inter-element coupling were not modeled in these works, salient features of the metasurface antennas and their performance in the context of microwave imaging could be captured. Only recently has the full analytical treatment of metasurface antennas based on coupled dipoles [117] become available. In this methodology, the metasurface is replaced by an array of coupled dipoles, whose responses are determined by solving a matrix equation that entails the interaction of elements through appropriate Green's functions [118]-[121]. 
These techniques open up the possibility of rapid and accurate modeling of illumination patterns generated by extremely large apertures. Using this capability, for example, we can implement DMAs that can generate patterns optimized for a specific imaging application [122]. Furthermore, we can use these analytical treatment to co-optimize the imaging hardware in the context of task-based classification [123], [124].

\section{E. Polarimetric imaging}

In all previous sections, only the reflectivity of the region to be imaged was interrogated by the systems, thus considering a scalar approximation of the interaction of the transmit and receive fields with the targets. It has been demonstrated in [125], [126] that the design of suitable metasurfaces can be modified to enable polarimetric imaging, even in close range. Compared to conventional antenna array architectures, this approach achieves the performance of a polarimetric and single-shot MIMO system. A proof of concept was carried out in [126] by designing a frequency-diverse cavity-backed metasurface operating in the $18-26 \mathrm{GHz}$ band. This device exploited radiation diversity in transmission and reception of pseudo-orthogonal fields in frequency, space, and polarization to ensure possible reconstruction of the susceptibility tensors at any location within a volume of interest (see Fig. 17).

The advantage of such an approach lies in the possibility of reconstructing all the elements of the susceptibility tensor at each position of space, unlike most polarimetric systems that only probe the components orthogonal to the propagation axis [127]. This improvement is based on the development of systems including radiating apertures whose dimensions are of the same order of magnitude as the distance from the targets to be imaged. In this configuration, the absence of a paraxial approximation gives access to additional information for the identification of targets in the region of interest. For each voxel of the domain to be imaged, it is thus possible to reconstruct all the complex components of a symmetric matrix. An example of a reconstruction made from a "D"-shaped target made of copper wire is shown in Fig. 17. This example highlights the significant contribution of the tensor components projected along the projection axis in range as well as the cross-range. In addition, unlike similar domains such as diffusion tensor imaging [128], the $3 \times 3$ tensor interrogated at each position in space is complex, significantly increasing the quantity of information that can be extracted from these estimates.

\section{F. Passive imaging and synthetic aperture interferometry}

Fusion of CI concepts and metamaterial antennas can also improve passive and interferometric imaging systems. In this context, it is not possible to control the source of electromagnetic radiation and the systems are only used in reception. In order to overcome these limitations, systems composed of multiple receivers are developed where the correlation of all received signals can be processed to extract information relating to the domain to be imaged. These systems are of particular interest in applications related to security screening [130]-[132], exploiting ambient electromagnetic radiation to produce images that reveal information that is invisible at

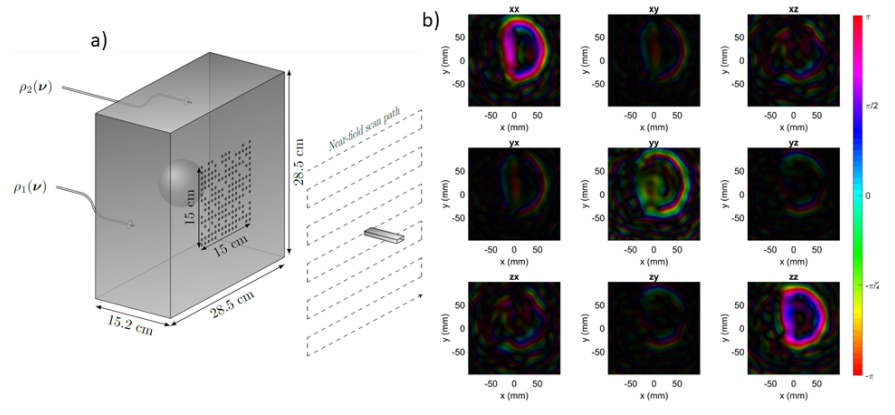

Fig. 17. a) Polarimetric computational imaging system. Each port of this leaky cavity allows secondary sources to be excited on its front panel. b) Reconstruction of susceptibility tensors [125]: A slice is extracted at the range of the target, made from a bent copper wire to form the letter $\mathrm{D}$. The radiating aperture is set in the plane $y=0$. The opacity of the pixels corresponds to the linear magnitude of the tensor. The phase is color-coded (from [125]).

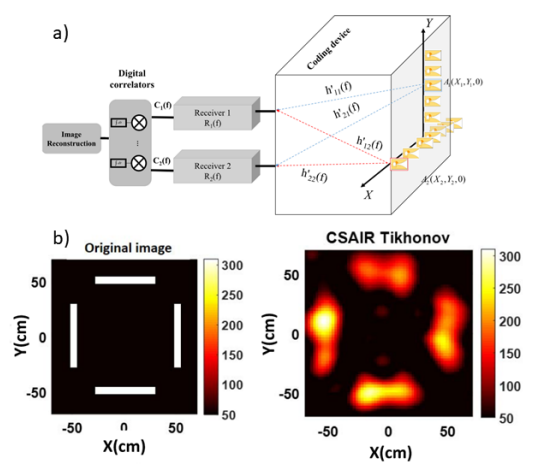

Fig. 18. a) A microwave interferometry system based on the use of a leaky cavity $\left(1 \times 1 \times 1 \mathrm{~m}^{3}\right)$. b) Experimental imaging results of distributed sources with a CSAIR architecture with 16 antennas and 4 RF chains. The signals are generated by a set of uncorrelated sources with thermal radiation in the 2-4 $\mathrm{GHz}$ band equivalent to that of a $300 \mathrm{~K}$ blackbody (from [129]).

optical wavelengths. The associated active architectures are, in this context too, particularly constrained by the redundancy of active systems necessary for the simultaneous acquisition of received signals, whether they are reflected by targets or directly emitted by their thermal radiation. It has recently been demonstrated that new architectures make it possible to greatly limit the number of acquisition chains by carrying out a physical multiplexing operation exploiting spatial and frequency diversity. These approaches can be based on the development of leaky cavities as shown in Fig. 18 [129], [133]-[135] as well as dispersive reflectarrays [136].

\section{CONCLUSION}

This article reviewed the fusion of metasurface antennas and computational imaging and development of novel microwave imaging systems. The physical operation of these systems were illustrated, and trade-offs governing their design were described. It illustrated steps taken to revamp metamaterial antenna hardware to match requirements of novel acquisition and processing techniques. The radical changes in the hardware and software when co-designing them, and the massive advantage of such an approach, was illustrated. We hope this review can serve as point of reference or inspiration in 
developing metasurface antennas for next-generation imaging, sensing, communication, and surveillance systems.

\section{ACKNOWLEDGMENT}

This work was supported by the Air Force Office of Scientific Research (AFOSR) (FA9550-18-1-0187).

\section{REFERENCES}

[1] D. M. Sheen, D. L. McMakin, and T. E. Hall, "Three-dimensional millimeter-wave imaging for concealed weapon detection," IEEE Trans. Microw. Theory Techn., vol. 49, no. 9, pp. 1581-1592, 2001.

[2] F. T. Ulaby, R. K. Moore, and A. K. Fung, "Microwave remote sensing: Active and passive. volume 1-microwave remote sensing fundamentals and radiometry," 1981.

[3] M. I. Skolnik, "Radar handbook," 1970.

[4] M. Soumekh, Synthetic aperture radar signal processing. New York: Wiley, 1999, vol. 7.

[5] S. S. Ahmed, A. Schiessl, and L. P. Schmidt, "A novel fully electronic active real-time imager based on a planar multistatic sparse array," IEEE Tran. Microw. Theory Techn., vol. 59, no. 12, pp. 3567-3576, Dec 2011.

[6] S. S. Ahmed, A. Schiessl, F. Gumbmann, M. Tiebout, S. Methfessel, and L. P. Schmidt, "Advanced microwave imaging," IEEE Microwave Magazine, vol. 13, no. 6, pp. 26-43, Sept 2012.

[7] M. T. Ghasr, M. Abou-Khousa, S. Kharkovsky, R. Zoughi, D. Pommerenke et al., "Portable real-time microwave camera at 24 ghz," IEEE Trans. Antennas Propag., vol. 60, no. 2, pp. 1114-1125, 2012.

[8] S. S. Ahmed, "Electronic microwave imaging with planar multistatic arrays," 2014.

[9] B. Gonzalez-Valdes, G. Allan, Y. Rodriguez-Vaqueiro, Y. Alvarez et al., "Sparse array optimization using simulated annealing and compressed sensing for near-field millimeter wave imaging," IEEE Trans. Antennas Propag., vol. 62, no. 4, pp. 1716-1722, 2014.

[10] W. F. Moulder, J. D. Krieger, J. J. Majewski, C. M. Coldwell, H. T. Nguyen et al., "Development of a high-throughput microwave imaging system for concealed weapons detection," in 2016 IEEE International Symposium on Phased Array Systems and Technology, 2016, pp. 1-6.

[11] G. Krieger, "Mimo-sar: Opportunities and pitfalls," IEEE Tran. on Geoscience and Remote Sensing, vol. 52, no. 5, pp. 2628-2645, 2014.

[12] R. C. Hansen, Phased array antennas. John Wiley \& Sons, 2009.

[13] M. Johnson, "Opening satellite capacity to consumers with metamaterial antennas," in Proceedings of the International Conference on Metamaterials, Photonic Crystals and Plasmonics, 2016.

[14] R. Stevenson, M. Sazegar, A. Bily, M. Johnson, and N. Kundtz, "Metamaterial surface antenna technology: Commercialization through diffractive metamaterials and liquid crystal display manufacturing," in 2016 International Congress on Advanced Electromagnetic Materials in Microwaves and Optics (METAMATERIALS). IEEE, pp. 349-351.

[15] D. L. Donoho, "Compressed sensing," Information Theory, IEEE Tran. on, vol. 52, no. 4, pp. 1289-1306, 2006.

[16] D. J. Brady, Optical imaging and spectroscopy. John Wiley \& Sons, 2009.

[17] J. N. Mait, G. W. Euliss, and R. A. Athale, "Computational imaging," Advances in Optics and Photonics, vol. 10, no. 2, pp. 409-483, 2018.

[18] N. Antipa, G. Kuo, R. Heckel, B. Mildenhall, E. Bostan, R. Ng, and L. Waller, "Diffusercam: lensless single-exposure 3d imaging," Optica, vol. 5, no. 1, pp. 1-9, 2018.

[19] A. Liutkus, D. Martina, S. Popoff, G. Chardon, O. Katz, G. Lerosey, S. Gigan, L. Daudet, and I. Carron, "Imaging with nature: Compressive imaging using a multiply scattering medium," Sci. Rep., vol. 4, 2014.

[20] C. M. Watts, D. Shrekenhamer, J. Montoya, G. Lipworth, J. Hunt T. Sleasman, S. Krishna, D. R. Smith, and W. J. Padilla, "Terahertz compressive imaging with metamaterial spatial light modulators," $\mathrm{Na}$ ture Photonics, 2014.

[21] G. Zheng, R. Horstmeyer, and C. Yang, "Wide-field, high-resolution fourier ptychographic microscopy," Nature photonics, vol. 7, no. 9, p. 739, 2013

[22] I. M. Vellekoop and A. Mosk, "Focusing coherent light through opaque strongly scattering media," Optics letters, vol. 32, no. 16, pp. 2309 2311, 2007.

[23] S. Popoff, G. Lerosey, R. Carminati, M. Fink, A. Boccara, and S. Gigan, "Measuring the transmission matrix in optics: an approach to the study and control of light propag. in disordered media," Physical review letters, vol. 104, no. 10, p. 100601, 2010.
[24] J. Bertolotti, E. G. Van Putten, C. Blum, A. Lagendijk, W. L. Vos, and A. P. Mosk, "Non-invasive imaging through opaque scattering layers," Nature, vol. 491, no. 7423, p. 232, 2012.

[25] O. Katz, P. Heidmann, M. Fink, and S. Gigan, "Non-invasive singleshot imaging through scattering layers and around corners via speckle correlations," Nature photonics, vol. 8, no. 10, p. 784, 2014.

[26] O. Katz, Y. Bromberg, and Y. Silberberg, "Compressive ghost imaging," Applied Physics Letters, vol. 95, no. 13, p. 131110, 2009.

[27] J. H. Shapiro, "Computational ghost imaging," Physical Review A, vol. 78, no. 6, p. 061802, 2008.

[28] J. Hunt, T. Driscoll, A. Mrozack, G. Lipworth, M. Reynolds, D. Brady, and D. R. Smith, "Metamaterial apertures for computational imaging," Science, vol. 339, no. 6117, pp. 310-313, 2013.

[29] X. Yang, Y. Pu, and D. Psaltis, "Imaging blood cells through scattering biological tissue using speckle scanning microscopy," Optics express, vol. 22, no. 3, pp. 3405-3413, 2014.

[30] I. M. Vellekoop, A. Lagendijk, and A. Mosk, "Exploiting disorder for perfect focusing," Nature photonics, vol. 4, no. 5, p. 320, 2010.

[31] M. F. Duarte, M. A. Davenport, D. Takhar, J. N. Laska, T. Sun, K. F. Kelly, and R. G. Baraniuk, "Single-pixel imaging via compressive sampling," IEEE signal process. magazine, vol. 25, no. 2, pp. 83-91, 2008 .

[32] L. C. Potter, E. Ertin, J. T. Parker, and M. Cetin, "Sparsity and compressed sensing in radar imaging," Proceedings of the IEEE, vol. 98, no. 6, pp. 1006-1020, 2010.

[33] V. M. Patel, G. R. Easley, D. M. Healy Jr, and R. Chellappa, "Compressed synthetic aperture radar," IEEE Journal of selected topics in signal processing, vol. 4, no. 2, pp. 244-254, 2010.

[34] N. Anselmi, M. Salucci, G. Oliveri, and A. Massa, "Wavelet-based compressive imaging of sparse targets," IEEE Tran. on Antennas and Propag., vol. 63, no. 11, pp. 4889-4900, 2015.

[35] S. Caorsi, A. Massa, and M. Pastorino, "A computational technique based on a real-coded genetic algorithm for microwave imaging purposes," IEEE Tran. on Geoscience and Remote Sensing, vol. 38, no. 4, pp. 1697-1708, Jul 2000.

[36] A. Massa, P. Rocca, and G. Oliveri, "Compressive sensing in electromagnetics-a review," IEEE Antennas and Propag. Magazine, vol. 57, no. 1, pp. 224-238, 2015.

[37] J. Helander, A. Ericsson, M. Gustafsson, T. Martin, D. Sjöberg, and C. Larsson, "Compressive sensing techniques for mm-wave nondestructive testing of composite panels," IEEE Tran. on Antennas and Propag. vol. 65, no. 10, pp. 5523-5531, 2017.

[38] H. Zamani and M. Fakharzadeh, "1.5-d sparse array for millimeterwave imaging based on compressive sensing techniques," IEEE Tran. on Antennas and Propag., vol. 66, no. 4, pp. 2008-2015, 2018.

[39] C. L. Holloway, E. F. Kuester, J. A. Gordon, J. O'Hara, J. Booth, and D. R. Smith, "An overview of the theory and applications of metasurfaces: The two-dimensional equivalents of metamaterials," IEEE Antennas and Propag. Magazine, vol. 54, no. 2, pp. 10-35, 2012.

[40] C. Pfeiffer and A. Grbic, "Metamaterial huygens' surfaces: tailoring wave fronts with reflectionless sheets," Physical review letters, vol. 110, no. 19, p. 197401,2013

[41] D. R. Jackson and A. A. Oliner, "Leaky-wave antennas," Modern Antenna Handbook, pp. 325-367, 2008.

[42] A. M. Patel and A. Grbic, "Modeling and analysis of printed-circuit tensor impedance surfaces," IEEE Tran. on Antennas and Propag., vol. 61, no. 1, pp. 211-220, 2013.

[43] S. Maci, G. Minatti, M. Casaletti, and M. Bosiljevac, "Metasurfing: Addressing waves on impenetrable metasurfaces," IEEE Antennas Wireless Propag. Letters, vol. 10, pp. 1499-1502, 2011.

[44] M. Nannetti, F. Caminita, and S. Maci, "Leaky-wave based interpretation of the radiation from holographic surfaces," in 2007 IEEE Antennas and Propag. Society International Symposium. IEEE, 2007, pp. 5813-5816.

[45] C. L. Holloway, A. Dienstfrey, E. F. Kuester, J. F. O’Hara, A. K. Azad, and A. J. Taylor, "A discussion on the interpretation and characterization of metafilms/metasurfaces: The two-dimensional equivalent of metamaterials," Metamaterials, vol. 3, no. 2, pp. 100-112, 2009.

[46] J. D. Baena, J. Bonache, F. Martin, R. M. Sillero, F. Falcone et al., "Equivalent-circuit models for split-ring resonators and complementary split-ring resonators coupled to planar transmission lines," IEEE Tran. on Microw. Theory Techn., vol. 53, no. 4, pp. 1451-1461, 2005.

[47] R. Liu, X. M. Yang, J. G. Gollub, J. J. Mock, T. J. Cui, and D. R. Smith, "Gradient index circuit by waveguided metamaterials," Applied Physics Letters, vol. 94, no. 7, p. 073506, 2009. 
[48] G. Lipworth, A. Mrozack, J. Hunt, D. L. Marks, T. Driscoll, D. Brady, and D. R. Smith, "Metamaterial apertures for coherent computational imaging on the physical layer," J. Opt. Soc. Amer. A, vol. 30, no. 8, pp. 1603-1612, 2013.

[49] A. D. Yaghjian, "An overview of near-field antenna measurements," IEEE Trans. Antennas Propag., vol. 34, no. 1, pp. 30-45, 1986.

[50] J. M. Bioucas-Dias and M. A. Figueiredo, "A new twist: Twostep iterative shrinkage/thresholding algorithms for image restoration," IEEE Tran. on Image processing, vol. 16, no. 12, pp. 2992-3004, 2007.

[51] M. Soumekh, "A system model and inversion for synthetic aperture radar imaging," IEEE Tran. on Image Processing, vol. 1, no. 1, pp. 64-76, 1992.

[52] — Fourier array imaging. Prentice-Hall, Inc., 1994.

[53] _ Synthetic aperture radar signal processing. New York: Wiley, 1999.

[54] O. Yurduseven, M. F. Imani, H. Odabasi, J. Gollub, G. Lipworth, A. Rose, and D. R. Smith, "Resolution of the frequency diverse metamaterial aperture imager," Progress In Electromagnetics Research, vol. 150, pp. 97-107, 2015.

[55] O. Yurduseven, P. Flowers, S. Ye, D. L. Marks, J. N. Gollub, T. Fromenteze, B. J. Wiley, and D. R. Smith, "Computational microwave imaging using $3 \mathrm{~d}$ printed conductive polymer frequency-diverse metasurface antennas," IET Microwaves, Antennas \& Propag., vol. 11, no. 14, pp. 1962-1969, 2017.

[56] A. W. Lohmann, R. G. Dorsch, D. Mendlovic, C. Ferreira, and Z. Zalevsky, "Space-bandwidth product of optical signals and systems," J. Opt. Soc. Amer. A, vol. 13, no. 3, pp. 470-473, 1996.

[57] H. Odabasi, M. F. Imani, G. Lipworth, J. Gollub, and D. R. Smith, "Investigation of alignment errors on multi-static microwave imaging based on frequency-diverse metamaterial apertures," Progress In Electromagnetics Research, vol. 70, pp. 101-112, 2016.

[58] O. Yurduseven, J. N. Gollub, K. P. Trofatter, D. L. Marks, A. Rose, and D. R. Smith, "Software calibration of a frequency-diverse, multistatic, computational imaging system," IEEE Access, vol. 4, pp. 2488-2497, 2016.

[59] A. Sharma, A. Pedross-Engel, D. Arnitz, C. M. Watts, D. R. Smith, and M. S. Reynolds, "A k-band backscatter fiducial for continuous calibration in coherent millimeter-wave imaging," IEEE Tran. Microw. Theory and Techn., vol. 66, no. 1, pp. 431-438, 2017.

[60] P. Fellgett, "On the ultimate sensitivity and practical performance of radiation detectors," JOSA, vol. 39, no. 11, pp. 970-976, 1949.

[61] M. E. Gehm, S. T. McCain, N. P. Pitsianis, D. J. Brady, P. Potuluri, and M. E. Sullivan, "Static two-dimensional aperture coding for multimodal, multiplex spectroscopy," Applied optics, vol. 45, no. 13, pp. 2965-2974, 2006.

[62] O. Furxhi, D. L. Marks, and D. J. Brady, "Echelle crossed grating millimeter wave beam scanner," Optics express, vol. 22, no. 13, pp. 16393-16407, 2014.

[63] J. Hunt, J. Gollub, T. Driscoll, G. Lipworth, A. Mrozack, M. S. Reynolds, D. J. Brady, and D. R. Smith, "Metamaterial microwave holographic imaging system," J. Opt. Soc. Amer. A, vol. 31, no. 10, pp. 2109-2119, 2014.

[64] G. Lipworth, A. Rose, O. Yurduseven, V. R. Gowda, M. F. Imani, H. Odabasi, P. Trofatter, J. Gollub, and D. R. Smith, "Comprehensive simulation platform for a metamaterial imaging system," Appl. Opt., vol. 54, no. 31, pp. 9343-9353, Nov 2015.

[65] G. Lipworth, N. W. Caira, S. Larouche, and D. R. Smith, "Phase and magnitude constrained metasurface holography at w-band frequencies," Optics Express, vol. 24, no. 17, pp. 19372-19387, 2016.

[66] A. D. Yaghjian and S. R. Best, "Impedance, bandwidth, and q of antennas," IEEE Tran. on Antennas and Propag., vol. 53, no. 4, pp. 1298-1324, 2005.

[67] T. Fromenteze, O. Yurduseven, M. F. Imani, J. Gollub, C. Decroze, D. Carsenat, and D. R. Smith, "Computational imaging using a modemixing cavity at microwave frequencies," Appl. Phys. Lett., vol. 106, no. 19 , p. 194104,2015

[68] G. Montaldo, D. Palacio, M. Tanter, and M. Fink, "Building threedimensional images using a time-reversal chaotic cavity," IEEE Tran. on ultrasonics, ferroelectrics, and frequency control, vol. 52, no. 9, pp. $1489-1497,2005$

[69] O. Yurduseven, V. R. Gowda, J. N. Gollub, and D. R. Smith, "Multistatic microwave imaging with arrays of planar cavities," IET Microw., Antennas \& Propag., vol. 10, no. 11, pp. 1174-1181, 2016.

[70] _ - "Printed aperiodic cavity for computational and microwave imaging," IEEE Microw.Wireless Compon. Lett., vol. 26, no. 5, pp. 367-369, May 2016.
[71] M. F. Imani, T. Sleasman, J. N. Gollub, and D. R. Smith, "Analytical modeling of printed metasurface cavities for computational imaging," Journal of Applied Physics, vol. 120, no. 14, 2016.

[72] D. L. Marks, J. N. Gollub, and D. R. Smith, "Spatially resolving antenna arrays using frequency diversity," J. Opt. Soc. Amer. A, vol. 33, no. 5, pp. 899-912, 2016.

[73] O. Yurduseven, J. N. Gollub, D. L. Marks, and D. R. Smith, "Frequency-diverse microwave imaging using planar mills-cross cavity apertures," Opt. Exp., vol. 24, no. 8, pp. 8907-8925, 2016.

[74] J. Gollub, O. Yurduseven, K. P. Trofatter, M. F. Imani, H. Odabasi, T. Sleasman et al., "Large metasurface aperture for millimeter wave computational imaging at the human-scale," Sci. Rep., vol. 7, p. 42650, 2017.

[75] O. Yurduseven, J. N. Gollub, A. Rose, D. L. Marks, and D. R. Smith "Design and simulation of a frequency-diverse aperture for imaging of human-scale targets," IEEE Access, vol. 4, pp. 5436-5451, 2016.

[76] A. Pedross-Engel, D. Arnitz, and M. S. Reynolds, "Self-jamming mitigation via coding for millimeter-wave imaging with direct conversion receivers," IEEE Microwave and Wireless Components Letters, vol. 27, no. 4, pp. 410-412, 2017.

[77] A. Pedross-Engel, D. Arnitz, J. N. Gollub, O. Yurduseven, K. P. Trofatter, M. F. Imani, T. Sleasman, M. Boyarsky, X. Fu, D. L. Marks et al., "Orthogonal coded active illumination for millimeter wave, massivemimo computational imaging with metasurface antennas," IEEE Tran. on Computational Imaging, vol. 4, no. 2, pp. 184-193, 2018.

[78] D. L. Marks, O. Yurduseven, and D. R. Smith, "Fourier accelerated multistatic imaging: a fast reconstruction algorithm for multiple-inputmultiple-output radar imaging," IEEE Access, vol. 5, pp. 1796-1809, 2017.

[79] — - "Near-field multistatic radar reconstruction with stretched-phase fourier accelerated multistatic imaging," IET Radar, Sonar \& Navigation, vol. 11, no. 11, pp. 1718-1729, 2017.

[80] T. Sleasman, M. Boyarsky, M. Imani, J. Gollub, and D. Smith, "Design considerations for a dynamic metamaterial aperture for computational imaging at microwave frequencies," JOSA B, vol. 33, no. 6, pp. 1098$1111,2016$.

[81] D. Sievenpiper, J. Schaffner, R. Loo, G. Tangonan, S. Ontiveros, and R. Harold, "A tunable impedance surface performing as a reconfigurable beam steering reflector," IEEE Tran. on antennas and Propag., vol. 50, no. 3, pp. 384-390, 2002.

[82] S. Lim, C. Caloz, and T. Itoh, "Metamaterial-based electronically controlled transmission-line structure as a novel leaky-wave antenna with tunable radiation angle and beamwidth," IEEE Tran. on Microwave Theory and Techniques, vol. 52, no. 12, pp. 2678-2690, 2004.

[83] D. J. Gregoire, J. S. Colburn, A. M. Patel, R. Quarfoth, and D. Sievenpiper, "A low profile electronically-steerable artificialimpedance-surface antenna," in Electromagnetics in Advanced Applications (ICEAA), International Conference on, Aug 2014, pp. 477-479.

[84] R. Guzman-Quiros, J. L. Gomez-Tornero, A. R. Weily, and Y. J. Guo, "Electronic full-space scanning with 1-d fabry-pérot lwa using electromagnetic band-gap," IEEE Antennas Wireless Propag. Letters, vol. 11, pp. 1426-1429, 2012.

[85] D. J. Gregoire, A. Patel, and R. Quarfoth, "A design for an electronically-steerable holographic antenna with polarization control," in IEEE APS and USNC/URSI National Radio Science Meeting, July 2015, pp. 2203-2204.

[86] J.-h. Fu, A. Li, W. Chen, B. Lv, Z. Wang, P. Li, and Q. Wu, "An electrically controlled crlh-inspired circularly polarized leaky-wave antenna," IEEE Antennas Wireless Propag. Letters, vol. 16, pp. 760763, 2017.

[87] K. Chen, Y. H. Zhang, S. Y. He, H. T. Chen, and G. Q. Zhu, "An electronically controlled leaky-wave antenna based on corrugated siw structure with fixed-frequency beam scanning," IEEE Antennas Wireless Propag. Letters, vol. 18, no. 3, pp. 551-555, 2019.

[88] T. Sleasman, M. F. Imani, W. Xu, J. Hunt, T. Driscoll, M. S. Reynolds, and D. R. Smith, "Waveguide-fed tunable metamaterial element for dynamic apertures," IEEE Antennas Wireless Propag. Lett., vol. 15, pp. 606-609, 2016.

[89] T. Sleasman, M. F. Imani, J. N. Gollub, and D. R. Smith, "Dynamic metamaterial aperture for microwave imaging," Applied Physics Letters, vol. 107, no. 20, p. 204104, 2015.

[90] J. M. Lopez-Sanchez and J. Fortuny-Guasch, "3-d radar imaging using range migration techniques," IEEE Tran. Antennas Propag., vol. 48 , no. 5, pp. $728-737,2000$

[91] X. Zhuge and A. Yarovoy, "Three-dimensional near-field mimo array imaging using range migration techniques," IEEE Tran. on Image Processing, vol. 21, no. 6, pp. 3026-3033, 2012. 
[92] L. Pulido-Mancera, T. Fromenteze, T. Sleasman, M. Boyarsky, M. F. Imani, M. Reynolds, and D. Smith, "Application of range migration algorithms to imaging with a dynamic metasurface antenna," JOSA $B$, vol. 33, no. 10, pp. 2082-2092, 2016.

[93] A. V. Diebold, L. Pulido-Mancera, T. Sleasman, M. Boyarsky, M. F. Imani, and D. R. Smith, "Generalized range migration algorithm for synthetic aperture radar image reconstruction of metasurface antenna measurements," JOSA B, vol. 34, no. 12, pp. 2610-2623, 2017.

[94] T. Fromenteze, M. Boyarsky, J. Gollub, T. Sleasman, M. Imani, and D. R. Smith, "Single-frequency near-field mimo imaging," in Antennas and Propag. (EUCAP), 2017 European Conference on, pp. 1415-1418.

[95] T. Sleasman, M. Boyarsky, M. F. Imani, T. Fromenteze, J. N. Gollub, and D. R. Smith, "Single-frequency microwave imaging with dynamic metasurface apertures," JOSA B, vol. 34, no. 8, pp. 1713-1726, 2017.

[96] M. Boyarsky, T. Sleasman, L. Pulido-Mancera, A. V. Diebold, M. F. Imani, and D. R. Smith, "Single-frequency 3d synthetic aperture imaging with dynamic metasurface antennas," Applied optics, vol. 57, no. 15, pp. 4123-4134, 2018.

[97] O. Yurduseven, T. Fromenteze, D. L. Marks, J. N. Gollub, and D. R. Smith, "Frequency-diverse computational microwave phaseless imaging," IEEE Antennas Wireless Propag. Lett., vol. 16, pp. 2808$2811,2017$.

[98] T. Fromenteze, X. Liu, M. Boyarsky, J. Gollub, and D. R. Smith, "Phaseless computational imaging with a radiating metasurface," $\mathrm{OP}$ tics express, vol. 24, no. 15, pp. 16760-16776, 2016.

[99] O. Yurduseven, T. Fromenteze, and D. R. Smith, "Relaxation of alignment errors and phase calibration in computational frequencydiverse imaging using phase retrieval," IEEE Access, vol. 6, pp. 14884 14 894, 2018.

[100] A. V. Diebold, M. F. Imani, T. Sleasman, and D. R. Smith, "Phaseless computational ghost imaging at microwave frequencies using a dynamic metasurface aperture," Applied Optics, vol. 57, no. 9, pp. 21422149, 2018.

[101] _ - "Phaseless coherent and incoherent microwave ghost imaging with dynamic metasurface apertures," Optica, vol. 5, no. 12, pp. 15291541, 2018.

[102] O. Yurduseven, D. L. Marks, T. Fromenteze, J. N. Gollub, and D. R. Smith, "Millimeter-wave spotlight imager using dynamic holographic metasurface antennas," Optics express, vol. 25 , no. 15 , pp. 18230 $18249,2017$.

[103] S. Gazit, A. Szameit, Y. C. Eldar, and M. Segev, "Super-resolution and reconstruction of sparse sub-wavelength images," Optics express, vol. 17 , no. 26, pp. 23920-23946, 2009.

[104] A. M. Maiden, M. J. Humphry, F. Zhang, and J. M. Rodenburg, "Superresolution imaging via ptychography," JOSA A, vol. 28, no. 4, pp. 604-612, 2011.

[105] M. Johnson, P. Bowen, N. Kundtz, and A. Bily, "Discrete-dipole approximation model for control and optimization of a holographic metamaterial antenna," App. Opt., vol. 53, no. 25, pp. 5791-5799, 2014.

[106] M. C. Johnson, S. L. Brunton, N. B. Kundtz, and J. N. Kutz, "Sidelobe canceling for reconfigurable holographic metamaterial antenna," IEEE Tran. Antennas and Propag., vol. 63, no. 4, pp. 1881-1886, April 2015.

[107] M. Boyarsky, T. Sleasman, L. Pulido-Mancera, T. Fromenteze, A. Pedross-Engel, C. M. Watts, M. F. Imani, M. S. Reynolds, and D. R. Smith, "Synthetic aperture radar with dynamic metasurface antennas: a conceptual development," JOSA A, vol. 34, no. 5, pp. A22-A36, 2017.

[108] C. M. Watts, A. Pedross-Engel, D. R. Smith, and M. S. Reynolds, "X-band sar imaging with a liquid-crystal-based dynamic metasurface antenna," JOSA B, vol. 34, no. 2, pp. 300-306, 2017.

[109] D. Li, X. Li, Y. Qin, Y. Cheng, and H. Wang, "Radar coincidence imaging: an instantaneous imaging technique with stochastic signals," IEEE Trans. Geosci. Remote Sens., vol. 52, no. 4, pp. 2261-2277, 2014.

[110] A. V. Diebold, M. F. Imani, and D. R. Smith, "Phaseless radar coincidence imaging with a mimo sar platform," Remote Sensing, vol. 11 , no. 5 , p. $533,2019$.

[111] A. Pedross-Engel, C. M. Watts, D. R. Smith, and M. S. Reynolds, "Enhanced resolution stripmap mode using dynamic metasurface antennas," IEEE Trans. Geosci. Remote Sens., vol. 55, no. 7, pp. 3764 3772, 2017.

[112] T. Sleasman, M. Boyarsky, L. Pulido-Mancera, T. Fromenteze, M. F. Imani, M. S. Reynolds, and D. R. Smith, "Experimental synthetic aperture radar with dynamic metasurfaces," IEEE Tran. on Antennas and Propag., vol. 65, no. 12, pp. 6864-6877, 2017.

[113] T. Sleasman, M. F. Imani, J. N. Gollub, and D. R. Smith, "Microwave imaging using a disordered cavity with a dynamically tunable impedance surface," Physical Review Applied, 2016.
[114] D. F. Sievenpiper, J. H. Schaffner, H. J. Song, R. Y. Loo, and G. Tangonan, "Two-dimensional beam steering using an electrically tunable impedance surface," IEEE Tran. on Antennas and Propag., vol. 51, no. 10, pp. 2713-2722, 2003.

[115] M. F. Imani, T. Sleasman, and D. R. Smith, "Two-dimensional dynamic metasurface apertures for computational microwave imaging," IEEE Antennas Wireless Propag. Lett., vol. 17, no. 12, pp. 2299-2303, 2018.

[116] M. Boyarsky, M. F. Imani, and D. R. Smith, "Grating lobe suppression in metasurface antenna arrays with a waveguide feed layer," arXiv preprint arXiv: 1905.09846, 2019.

[117] B. T. Draine and P. J. Flatau, "Discrete-dipole approximation for scattering calculations," JOSA A, vol. 11, no. 4, pp. 1491-1499, 1994.

[118] L. M. Pulido-Mancera, T. Zvolensky, M. F. Imani, P. T. Bowen, M. Valayil, and D. R. Smith, "Discrete dipole approximation applied to highly directive slotted waveguide antennas," IEEE Antennas Wireless Propag. Letters, vol. 15, pp. 1823-1826, 2016.

[119] L. Pulido-Mancera, P. T. Bowen, M. F. Imani, N. Kundtz, and D. Smith, "Polarizability extraction of complementary metamaterial elements in waveguides for aperture modeling," Physical Review B, vol. 96, no. 23, p. 235402, 2017.

[120] L. Pulido-Mancera, M. F. Imani, P. T. Bowen, N. Kundtz, and D. R. Smith, "Analytical modeling of a two-dimensional waveguide-fed metasurface," arXiv preprint arXiv:1807.11592, 2018.

[121] I. Yoo, M. F. Imani, L. Pulido-Mancera, T. Sleasman, and D. R. Smith, "Analytic model of a coax-fed planar cavity-backed metasurface antenna for pattern synthesis," IEEE Tran. Antennas Propag., vol. 67, no. 9, pp. 5853-5866, 2019.

[122] M. Liang, Y. Li, H. Meng, M. Neifeld, and H. Xin, "Reconfigurable array design to realize principal component analysis (pca)-based microwave compressive sensing imaging system," Antennas Wireless Propag. Letters, IEEE, vol. 14, pp. 1039-1042, 2015.

[123] P. del Hougne, M. F. Imani, A. V. Diebold, R. Horstmeyer, and D. R. Smith, "Artificial neural network with physical dynamic metasurface layer for optimal sensing," arXiv preprint arXiv:1906.10251, 2019.

[124] L. Li, Y. Shuang, Q. Ma, H. Li, H. Zhao, M. Wei, C. Liu, C. Hao, C.W. Qiu, and T. J. Cui, "Intelligent metasurface imager and recognizer," Light: Science \& Applications, vol. 8, no. 1, pp. 1-9, 2019.

[125] T. Fromenteze, O. Yurduseven, M. Boyarsky, J. Gollub, D. L. Marks, and D. R. Smith, "Computational polarimetric microwave imaging," Optics express, vol. 25, no. 22, pp. $27488-27505,2017$.

[126] T. Fromenteze, M. Boyarsky, O. Yurduseven, J. Gollub, D. L. Marks, and D. R. Smith, "Computational polarimetric localization with a radiating metasurface," in 2017 IEEE International Symposium on Antennas and Propag., 2017, pp. 407-408.

[127] J. Wang, P. Aubry, and A. Yarovoy, "A novel approach to fullpolarimetric short-range imaging with copolarized data," IEEE Tran. on Antennas and Propag., vol. 64, no. 11, pp. 4733-4744, 2016.

[128] D. Le Bihan, J.-F. Mangin, C. Poupon, C. A. Clark, S. Pappata, N. Molko, and H. Chabriat, "Diffusion tensor imaging: concepts and applications," Journal of Magnetic Resonance Imaging, vol. 13, no. 4 pp. 534-546, 2001.

[129] E. Kpré, C. Decroze, M. Mouhamadou, and T. Fromenteze, "Computational imaging for compressive synthetic aperture interferometric radiometer," IEEE Tran. on Antennas and Propag., vol. 66, no. 10, pp. 5546-5557, 2018.

[130] L. Yujiri, M. Shoucri, and P. Moffa, "Passive millimeter wave imaging," IEEE microwave magazine, vol. 4, no. 3, pp. 39-50, 2003.

[131] M. Peichl, S. Dill, M. Jirousek, and H. Suess, "Passive microwave remote sensing for security applications," in 2007 European Radar Conference, pp. 32-35.

[132] R. Appleby and R. N. Anderton, "Millimeter-wave and submillimeterwave imaging for security and surveillance," Proceedings of the IEEE, vol. 95, no. 8, pp. 1683-1690, 2007.

[133] E. L. Kpré and C. Decroze, "Passive coding technique applied to synthetic aperture interferometric radiometer," IEEE Geoscience and Remote Sensing Letters, vol. 14, no. 8, pp. 1193-1197, 2017.

[134] — , "Synthetic aperture interferometric imaging using a passive microwave coding device," in Antenna Measurements \& Applications (CAMA), 2016 IEEE Conference on. IEEE, 2016, pp. 1-4.

[135] A. C. Tondo Yoya, B. Fuchs, and M. Davy, "Computational passive imaging of thermal sources with a leaky chaotic cavity," Applied Physics Letters, vol. 111, no. 19, p. 193501, 2017.

[136] A. Molaei, J. H. Juesas, W. J. Blackwell, and J. A. Martinez-Lorenzo, "Interferometric sounding using a metamaterial-based compressive reflector antenna," IEEE Tran. on Antennas and Propag., vol. 66, no. 5, pp. $2188-2198,2018$ 